IRA-International Journal of Management \&

Social Sciences

ISSN 2455-2267; Vol.09, Issue 03 (December 2017)

Pg. no. 86-125

Institute of Research Advances

https://research-advances.org/index.php/RAJMSS

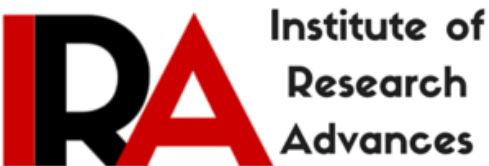

\title{
Accelerator and Multiplier for Macroeconomic Processes with Memory
}

\author{
Valentina V. Tarasova1, Vasily E. Tarasov ${ }^{2 \#}$ \\ ${ }^{1}$ Lomonosov Moscow State University Business School, Lomonosov Moscow State University, Moscow 119991, \\ Russian Federation. \\ ${ }^{2}$ Skobeltsyn Institute of Nuclear Physics, Lomonosov Moscow State University, Moscow 119991, Russian Federation.
}

\#corresponding author.

Type of Review: Peer Reviewed.

DOI: http://dx.doi.org/10.21013/jmss.v9.v3.p1

How to cite this paper:

Tarasova, V.V., Tarasov, V.E. (2017). Accelerator and Multiplier for Macroeconomic Processes with Memory. IRA-International Journal of Management \& Social Sciences (ISSN 2455-2267), 9(3), 86-125. doi:http://dx.doi.org/jmss.v9.v3.p1

(C) Valentina V. Tarasova \& Vasily E. Tarasov.

\section{(cc) BY-NC}

This work is licensed under a Creative Commons Attribution-Non Commercial 4.0 International License subject to proper citation to the publication source of the work.

Disclaimer: The scholarly papers as reviewed and published by the Institute of Research Advances (IRA) are the views and opinions of their respective authors and are not the views or opinions of the IRA. The IRA disclaims of any harm or loss caused due to the published content to any party.

Institute of Research Advances is an institutional publisher member of Publishers Inter Linking Association Inc. (PILA-CrossRef), USA. The institute is an institutional signatory to the Budapest Open Access Initiative, Hungary advocating the open access of scientific and scholarly knowledge. The Institute is a registered content provider under Open Access Initiative Protocol for Metadata Harvesting (OAI-PMH).

The journal is indexed \& included in WorldCat Discovery Service (USA), CrossRef Metadata Search (USA), WorldCat (USA), OCLC (USA), Open J-Gate (India), EZB (Germany) Scilit (Switzerland), Airiti (China), Bielefeld Academic Search Engine (BASE) of Bielefeld University, Germany, PKP Index of Simon Fraser University, Canada. 


\begin{abstract}
The paper proposes an approach to the description of macroeconomic phenomena, which takes into account the effects of fading memory. The standard notions of the accelerator and the multiplier are very limited, since the memory of economic agents is neglected. We consider the methods to describe the economic processes with memory, which is characterized by the fading of a power-law type. Using the mathematical tools of derivatives and integrals of non-integer orders, we suggest a generalization of the concept of the accelerator and multiplier. We derive the equations of the accelerator with memory and the multiplier with memory, which take into account the changes of endogenous and exogenous variables on a finite time interval. We prove the duality of the concepts of the multiplier with memory and the accelerator with memory. The proposed generalization includes the standard concepts of the accelerator and the multiplier as special cases. In addition these generalizations provide a range of intermediate characteristics to take into account the memory effects in macroeconomic models.
\end{abstract}

Keywords: macroeconomics; multiplier; accelerator; dynamic memory; memory effect; fractional derivatives; fractional integrals; multiplier with memory; accelerator with memory; duality

JEL C02 Mathematical Methods

JEL E00 - Macroeconomics and Monetary Economics: General

MSC 91B02 Fundamental topics (basic mathematics, applicable to economics in general)

MSC 91B55 Economic dynamics

MSC 26A33 Fractional derivatives and integrals

\title{
1. Introduction
}

Macroeconomics studies the functioning of national, regional and global economic systems as a whole. In the framework of macroeconomics, different models are constructed to explain the relationship between national income, production, consumption, saving, investment, employment, inflation, and other factors and indicators. The founder of modern macroeconomic theory is John Maynard Keynes, who published his monograph "The general theory of employment, interest and money" in 1936 (see Keynes, J. M. (1936, 2012)). The "Keynesian Revolution" and the birth of modern macroeconomics occurred almost simultaneously with the appearance of concepts of multiplier and accelerator, which were actively used in various models of economic growth. The "Marginal Revolution" and the "Keynesian Revolution" introduced fundamental economic concepts into economic analysis, which led to the use of the mathematical tools based on the derivatives and integrals of integer orders, the differential and finite difference equations (for example, see Samko, S. G. et. al. (1993), Kiryakova, V. S. (1994), Podlubny, I. (1998), Nakhushev, A. M. (2003), Kilbas, A. A., et. al. (2006), Diethelm, K. (2010)).

In macroeconomic models various simplifying assumptions are used to describe the economic processes. Because of this, many economic models have disadvantages, since they do not take into account important aspects of economic phenomena and processes. Some of these disadvantages are partially caused by limitations of the used mathematical tools. It is known that the derivatives of positive integer orders are determined by the properties of the differentiable function only in an infinitesimal neighborhood of the considered point. As a result, differential equations with derivatives of integer orders with respect to time cannot describe processes with memory, Tarasova, V. V. and Tarasov, V. E. (2018a). In fact, such equations describe only economic processes and systems, in which all economic agents remember only the infinitesimal past and the amnesia occurs infinitely fast. Differential equations with derivatives of integer orders describe the behavior of only those economic agents that suffer from amnesia. It is obvious that the assumption of the absence of memory in economic agents is a strong restriction. In this paper, we propose generalizations of the concepts of the accelerator and the multiplier, which allow us to take into account the effects of memory in macroeconomic models.

\section{Multiplier with Memory}

\subsection{Concept of Standard Multiplier}

The description of macroeconomic processes is realized by using models, Allen, R. G. D. (1959, 1968). In these models, two types of variables are used: the exogenous variables (factors) and the endogenous variables (indicators). The exogenous variables are values that are defined as external for a given model, i.e., that are 
formed outside the model. These variables are treated as independent (autonomous) variables. The endogenous variables are internal variables of the model, i.e., the variables that are formed within the model. These variables are dependent quantities, which are described as functions of independent variables.

In macroeconomics, the multiplier describes how much an endogenous variable changes in response to a change of some exogenous variables. The multipliers show how much the final indicators will change with the change of various economic factors.

The notion a multiplier was proposed by Kahn, R. F. (1931). Using the employment multiplier (the Kahn's multiplier), he proved that government spending on organizing of the public works not only leads to increased employment, but also leads to an increase in consumer demand, and this, in turn, contributes to the growth of production and employment in other sectors of the economy. The increase in aggregate expenditure (for example government spending for "public works") can lead to the increase of output and income.

In 1936 John Keynes published the book "The General Theory of Employment, Interest and Money" (see Keynes, J. M. $(1936,2012)$ ), which has led to the Keynesian revolution in economic analysis and the birth of modern macroeconomic theory. In this work, Keynes developed the concept of an economic multiplier and multiplicative effects. He proposed the concepts of investment multiplier, saving and consumption multipliers. An investment multiplier (the Keynes multiplier) describes how much the income increases with increasing investment per unit. The multiplier of accumulation (or consumption) shows how much the accumulation (consumption) increases with an increase in income per unit.

If time is considered as a continuous parameter, the indicators and factors (exogenous and endogenous variables) are usually described by continuously differentiable functions of time t. Let us consider an endogenous variable $\mathrm{Y}(\mathrm{t})$, which depends on the exogenous variable $\mathrm{X}(\mathrm{t})$. If this dependence is linear, then the multiplier equation has the form

$Y(t)=m \cdot X(t)+b$,

where $m$ and $b$ are some constants. The number $\mathrm{m}$ is called the multiplier coefficient of the endogenous variable $\mathrm{Y}(\mathrm{t})$, and the free term $\mathrm{b}$ is interpreted as a part of this variable, which does not depend on the exogenous variable $\mathrm{X}(\mathrm{t})$, Allen, R. G. D. (1959), p. 59. In linear dependence, the increase of the factor X(t) by the value $\Delta \mathrm{X}(\mathrm{t})$ generates the increase of the indicator $\mathrm{Y}(\mathrm{t})$ by the quantity $\Delta Y(t)$, which is described be the direct proportionality $\Delta Y(t)=m \cdot \Delta X(t)$. In the general case (in the linear and nonlinear dependence of $\mathrm{Y}(\mathrm{t})$ on $\mathrm{X}(\mathrm{t}))$, the coefficient $m$ is equal to the marginal value, i.e., $m=M Y_{X}(t)=\frac{Y^{(1)}(t)}{X^{(1)}(t)}$, where $Y^{(1)}(t)$ and $X^{(1)}(t)$ are the derivatives of the continuously differentiable functions, and $X^{(1)}(t) \neq 0$. If the function $\mathrm{X}(\mathrm{t})$ is invertible in a neighborhood of $\mathrm{t}$, then there is a single-valued function $\mathrm{Y}=\mathrm{Y}(\mathrm{X})$, and $m=M Y_{X}=\frac{d Y(X)}{d X}$.

For example, the income $\mathrm{Y}(\mathrm{t})$ can be divided into the consumed and saving parts: the consumption $C(t)$ and the saving $S(t)$. The consumption multiplier determines the dependence of the consumption increment $\Delta C(t)$ through the income increment $\Delta Y(t)$ by the equation $\Delta C(t)=m \cdot \Delta Y(t)$, where $\mathrm{m}$ is the consumption multiplier coefficient. The coefficient $m$ is equal to the marginal propensity to consume $m=M C_{Y}=\frac{d C(Y)}{d Y=\frac{C^{(1)}(t)}{Y^{(1)}(t)}}$, where $Y^{(1)}(t) \neq 0$. In the case of the linear dependence we have the equation $C(t)=m \cdot Y(t)+b$, where the free term $b$ is interpreted as part of the consumption, which is not affected by the income. Parameter $b$ allows us to describe negative saving at low income levels, Allen, R. G. D. (1959), p. 59.

The equation of the investment multiplier $\Delta Y(t)=m \cdot \Delta I(t)$ describes the increment of income $\Delta Y(t)$, which is caused by the increment of investment $\Delta I(t)$, where $\mathrm{m}$ is the multiplier coefficient of investment. According to Keynes, this coefficient is equal to the inverse value of the marginal propensity to save ( $m=\frac{1}{M S_{Y}}$, where $\left.M S_{Y}=1-M C_{Y}\right)$. If the total amount of investments is increased, then the income increases by a certain amount, which is greater than the increase of investment. The concept of an investment multiplier allows us to describe the change in national income when changing government spending. Macroeconomics also uses a more general concept such an exogenous spending multiplier, which characterizes the change of the national income with a single change of the autonomous exogenous expenditures. 


\subsection{Multiplier Effect}

The effect of the multiplier of exogenous expenditures is the effect of the change (increase or decrease) of any component of autonomous expenditures, which leads to a change in the national income by an amount that is greater than the initial change of the expenditure. The autonomous expenditures are expenditures that are considered as independent of the level of income. The mechanism, which leads to the multiplier effect, is caused by the fact that the initial increase in exogenous expenditures may lead to an increase of the consumer spending, and therefore to an increase of the incomes of other consumers, and this leads to a further increase in consumption, etc. It leads to a general increase of the national income more than the initial value of expenditure. The effect of increasing of the endogenous variable is called the multiplier effect, when the multiplier coefficient is greater than one.

The mechanism of the appearance of the multiplier effect for investment is the following. The initial increase of the investment leads to an increase of the companies incomes. These companies, in turn, increase production and employment. This leads to additional demand for materials and other goods. The increase of the level of employment and income of the company employees leads to an increase in their consumption, and, as a result, to increased production and increased income in industries that create these goods and services. As a result, there is a chain of reaction of the income growth and the increased production, which covers an increasing number of industries.

The memory effect can significantly influence the multiplier effect mechanism. Let us explain the action of the memory effect on the multiplier mechanism. Economic agents can remember previous negative (or positive) changes in the economy, and as a consequence, in case of a repetition of the situation, the agents can behave differently. For example, the agents can increase or decrease the share of income channeled to saving and accumulation. In general, the propensity to save is directly related to the presence of the memory of economic agents, and, therefore, may depend on changes in income and consumption at previous points in time.

The process of appearance of the multiplier effect is often compared with the process of formation of circles on water after a primary disturbance caused by a stone thrown into the water. An analogy with the formation of circles and waves in the liquid medium can be continued. The appearance of the multiplier effects for economic processes with memory in a sense is analogous to the propagation of the initial impact (disturbances) and waves in continuous media with a memory (for example, see Lokshin, A. A., Suvorova, Yu. V. (1982), Mainardi, F. (2010)).

\subsection{Effects of Delay and Memory}

Usually economic models with continuous time are described by equations that contain only derivatives of positive integer orders with respect to time. It is known that such derivatives are determined by the properties of differentiable functions only in an infinitesimal neighborhood of the considered time point. Therefore, the differential equations with derivatives of integer orders actually describe only the instantaneous reaction of economic agents without memory. Such equations do not take into account that economic agents have a memory of the history of changes in indicators and factors on a time interval. It can be said that the standard equations of the multiplier and accelerator depend only on infinitesimal neighborhood of the considered point of time. Therefore these equations are applicable only if all economic agents have total amnesia. Obviously, this condition leads to greatly simplified macroeconomic models, and it cannot always be used to describe real economy. This restriction is mathematically conditioned by the use of mathematical tools of derivatives of integer orders.

Interrelations between economic agents, as a rule, are not instantaneous, i.e. they are not local in time. Between the causes and consequences, between the primary impact and its effect (for example, between investment and income generation), there is a period of time, called a time lag (delay). In real economic processes, temporary lags are present in most links. For example, in the modeling of economic dynamics, it is important to take into account an investment lag, which is caused by the finiteness of the period from the design of an object to its commissioning at full capacity. The absence of an instantaneous reaction of the indicator to changes of the determining factor means the time nonlocality of these links. In many economic processes, the lag is not a strictly defined quantity, but it is distributed in time. The macroeconomic models, which assume the absence of the time lags, are a strong simplification of reality. 
To take into account the time lag in macroeconomic models with continuous time, we can use the differential equations with derivatives of integer orders and retarded arguments (deviating arguments), Allen, R. G. D. (1959), Elsgolts, L. E., Norkin, S. B. (1973). This delay can be caused by various reasons. For example, it may be caused by the presence of inertia in some economic agents or the limited speed of economic processes.

However, economic models with delay do not take into account the memory effects. The distributed delay is not equivalent to the existence of memory in economic agents. It is necessary to distinguish the effects of time delay and dynamic memory. The effect of the time lag is related to the fact that all economic processes are realized with finite rates, i.e., the change of the economic factor does not lead to an instantaneous change of the indicator. The memory effect means that the state of the economy depends not only on the changes of the economic indicators and factors at a given time, but also on their values at previous points in time, Tarasova, V. V., Tarasov, V. E. (2018a). In general, the state of the economy at present time depends on the state changes at previous times. The memory effect is associated with the presence of memory in economic agents. It is caused by the fact that the economic process depends on previous changes of this process. The memory effect is manifested in the fact that the repetition of similar changes of the economic factor, gives the changes of the economic indicator by another way. This leads to the ambiguous (multivalued) dependencies of the indicator on the factor, Tarasova, V. V., Tarasov, V. E. (2017c). Multivalued dependencies of the indicator on the factor are caused by the fact that the economic agents remember the previous changes of this factor and the reaction of the considered indicator. Therefore, these agents can react differently to the same changes of the factor. As a result, in spite of the similar changes of the factor, the behavior of the indicator will be different.

The consideration of memory effects is important for describing real processes in the economy (for example, see Teyssiere, G., Kirman, A. P. (2007); Tarasov, V. E., Tarasova, V. V. (2016); Tarasova, V. V., Tarasov, V. E. (2018a)). Macroeconomic models with memory show that the effects of dynamic memory can essentially change the dynamics of economic growth (for details see Tarasova, V. V., Tarasov, V. E. (2016c,d,e,f g), (2017a,b,d,e), (2018b); Tarasov, V. E., Tarasova, V. V. (2017)).

Necessity to take into account dynamic memory in the economic process is caused by the fact that agents, which participate in this process, have a memory about the past of the process. As a result, to describe the economic process, we should take into account not only the current state and infinitely close to it previous states, but all the previous states of the considered process on a finite time interval. Mathematically, this means that to describe such a process it is not enough to use the state variables and their derivatives of integer orders with respect to time. In other words, the economic process with memory depends on the previous history of the process change. One of the first, who formulated the basic principle of processes with memory, was Volterra, V. (2005), Chapter VI, Section IV. This principle describes the memory fading (dissipation). In his book, Volterra, V. (2005), proposed to use integral equations to describe the processes with memory.

In modern mathematics, in addition to derivatives and integrals of integer orders, the different types of derivatives and integrals of non-integer (fractional) orders are known (see (for example, see Samko, S. G. et. al. (1993), Kiryakova, V. S. (1994), Podlubny, I. (1998), Nakhushev, A. M. (2003), Kilbas, A. A., et. al. (2006), Diethelm, K. (2010), Tarasova, V. V., Tarasov, V. E. (2017c)). These fractional operators have been suggested by Riemann, Liouville, Letnikov, Sonin, Weyl, Riesz, Erdelyi, Kober and Caputo. The derivatives and integrals of non-integer orders are actively used in the natural sciences to describe the processes with dynamic memory (for example see Mainardi, F. (2010), Tarasov, V. E. (2010)). To describe memory effects in economics, we can use equations with derivatives of non-integer orders, which are actually integro-differential operators, and fractional integrals of non-integer orders.

\subsection{Mathematical Description of Delay and Memory Effects}

For simplicity, we restrict ourselves to the consideration of linear dependencies, for which the equations of the multipliers are described by linear equations with continuous time. Let the variables $X(t)$ and $Y(t)$ be functions of time $t$ that describe the exogenous and endogenous variables respectively. For a linear dependence without delay, the multiplier equation has the form

$Y(t)=m \cdot X(t)+b$,

where $m$ and $b$ are some numerical coefficients. The number $m$ is called the multiplier coefficient of the endogenous variable $\mathrm{Y}(\mathrm{t})$, and the free term $b$ is interpreted as a part that does not depend on the exogenous variable $\mathrm{X}(\mathrm{t})$, Allen, R. G. D. (1959), p. 59. Equation (2.2) connects the sequence of subsequent states of the 
economic process with the previous states, through a single current state at each instant of time t. Therefore, it is said that equation (2.2) corresponds to economic processes without dynamic memory Tarasov, V. E. (2010), p. 394-395, Tarasova, V. V., Tarasov, V. E. (2018a).

In the simplest case of delay with a fixed duration, the multiplier equation (see Allen, R. G. D. (1959), p. 42) has the form

$Y(t)=m \cdot X(t-T)+b$,

where $\mathrm{T}$ is a positive constant that characterizes the time delay. In a more general case, the equation of multiplier with time delay, which has a fixed duration $\mathrm{T}$, is written in the form

$Y(t)=m \cdot \sum_{n=0}^{\infty} K_{n}(T) \cdot X(t-k \cdot T)+b$

where it is assumed that the coefficients $K_{n}(T)$ satisfy the normalization condition $\sum_{n=0}^{\infty} K_{n}(T)=1$.

The multiplier with continuously distributed delay (see Allen, R. G. D. (1959), p. 42), which is a continuous analog of formula (2.4), is described by the equation

$$
Y(t)=m \cdot \int_{0}^{\infty} K(\tau) \cdot X(t-\tau) d \tau+b,
$$

where $K(\tau)$ is a weighting function (see Allen, R. G. D. (1959), p. 25-26) that satisfies the normalization condition

$$
\int_{0}^{\infty} K(\tau) d \tau=1
$$

Since, under condition (2.6), the economic process, which is described by equation (2.5), passes through all states without any losses, we can say that equation (2.5) corresponds to a complete (perfect, ideal) memory, Tarasov, V. E. (2010), p. 394-395, Tarasova, V. V., Tarasov, V. E. (2018a)..

In the general case, normalization condition (2.6) is not necessary. If condition (2.6) is used, the coefficient $m$ of equation (2.5) is the multiplier coefficient. If equality (2.6) is not used as a condition on the function $K(\tau)$, then we can use the function $M(\tau)=m \cdot K(\tau)$ that plays the role of the generalized multiplier coefficient, and the coefficient $m$ can be removed from equation (2.5). Equation (2.5) is usually used to describe the distributed delay, and the function $K(\tau)$, characterizes the distribution of the delay in time. Using the function $M(\tau)=m \cdot K(\tau)$ and the change of variable $(t-\tau \rightarrow \tau)$, equation (2.5) can be rewritten in the form

$$
Y(t)=\int_{-\infty}^{t} M(t-\tau) \cdot X(\tau) d \tau+b .
$$

In equation (2.7), the endogenous variable $\mathrm{Y}(\mathrm{t})$ depends not only on the values of $\mathrm{X}(\tau)$ at one previous moment of time, as in equation (2.3) for time delay with a fixed duration. In equation (2.7), the indicator $\mathrm{Y}(\mathrm{t})$ depends on the values of the factor $\mathrm{X}(\tau)$ at all past instants on the infinite interval $(-\infty, t)$. The use of infinite limit in the integral of equation (2.7) means that we take into account the values of $\mathrm{X}(\tau)$ from an infinite distant past (actually from the birth of the Universe itself in the Big Bang). To describe the economic processes, we do not need to consider the infinitely distant past. For purposes of economic analysis, we can choose the initial time instant equal to zero in order to consider economic dynamics on a finite interval of the positive semi-axis. For this, we assume the function $M(\tau)$ to be zero for $\tau<0$. In this case, equation (2.7), we can write in the form

$$
Y(t)=\int_{0}^{t} M(t-\tau) \cdot X(\tau) d \tau+b .
$$

If the function $M(\tau)$ is expressed in terms of the Dirac delta-function by the formula $M(t-\tau)=m \cdot \delta(t-\tau)$, then equation (2.8) takes the form of equation (2.2), which describes the standard multiplier without delay. If $M(t-\tau)=m \cdot \delta(t-T-\tau)$, then equation (2.8) takes the form of equation (2.3) that describes the multiplier with delay, which has a fixed duration, Allen, R. G. D. (1959), p. 25. If $M(\tau)=m \cdot K(\tau)$, where $K(\tau)$ satisfies the normalization condition, then we get equation for the distributed delay.

As a result, equation (2.8) can be considered as a generalization of the standard multiplier. 


\subsection{Generalized Multiplier with Memory}

To describe economic processes with memory, it should be taken into account that the indicator $\mathrm{Y}(\mathrm{t})$ at time $\mathrm{t}$ depends not only on the value of the factor $\mathrm{X}(\mathrm{t})$ at the same time, but also on the changes $\mathrm{X}(\tau)$ on a finite time interval $0 \leq \tau \leq t$. The reason for this dependence is not directly connected with the finiteness of the speed of economic processes, which leads to the effects of distributed delay (time lag). The main reason to take into account the memory effects in economic processes is the fact that the economic agents remember the previous changes of the factor $\mathrm{X}(\mathrm{t})$ and their influence on changes of the indicator $\mathrm{Y}(\mathrm{t})$. The economic process is a processes with memory if there exists at least one endogenous variable $\mathrm{Y}(\mathrm{t})$ at the time $\mathrm{t}$, which depends on the history of the change of $\mathrm{X}(\tau)$ at $\tau \in(0, t)$. As a result, we get the following definition of the generalized multiplier with memory.

Definition. The generalized multiplier with memory is the dependence of an endogenous variable $Y(t)$ at the time $t \geq 0$ on the history of the change of the exogenous variable $X(\tau)$ on a finite time interval $0 \leq \tau \leq t$ such that $Y(t)=F_{0}^{t}(X(\tau))$

where $F_{0}^{t}$ denotes a certain rule which specifies values the value of $Y(t)$ for any time $t$, if $X(\tau)$ is known for $\tau \in[0, t]$. The operator $F_{0}^{t}$ transforms each history of changes of $X(\tau)$ for $\tau \in[0, t]$ into the corresponding history of changes of $Y(\tau)$ with $\tau \in[0, t]$.

The generalized multiplier with memory (2.9) is called linear, if the condition

$F_{0}^{t}\left(a \cdot X_{1}(\tau)+b \cdot X_{2}(\tau)\right)=a \cdot F_{0}^{t}\left(X_{1}(\tau)\right)+b \cdot F_{0}^{t}\left(X_{2}(\tau)\right)$

holds for all $\mathrm{a}$ and $\mathrm{b}$ from the field of scalars (for example, $\mathrm{a}$ and $\mathrm{b}$ are real numbers).

We can consider the linear operator $F_{0}^{t}$ in the form of the Volterra integral operator

$F_{0}^{t}(X(\tau)):=\int_{0}^{t} M(t, \tau) \cdot X(\tau) d \tau$

where the function $M(t, \tau)$ is the kernel of this integral operator. The function $M(t, \tau)$ is called the memory function. It allows us to define the linear generalized multiplier with memory of the special kind.

Definition. The generalized multiplier with memory (2.9) is called the linear generalized multiplier with memory function $M(t, \tau)$ if the multiplier (2.9) can be represented in the form

$Y(t)=\int_{0}^{t} M(t, \tau) \cdot X(\tau) d \tau$

where the function $M(t, \tau)$ describes the dynamic memory.

It is obvious that not every function $M(t, \tau)$ in equation (2.12) can be used to describe the economic multipliers with dynamic memory, Tarasova, V. V., Tarasov, V. E. (2018a). We will assume that expression (2.12) has a sense, i.e., we will assume that the integral (2.12) exists. Some properties of the multipliers with memory and some its examples will be considered in this paper below.

For the memory function $M(t, \tau)$, which is represented by the Dirac delta-function $(M(t, \tau)=m \cdot \delta(t-\tau))$, equation (2.12) has the form of the standard multiplier equation $Y(t)=m \cdot X(t)$. For the function $M(t, \tau)=m \cdot \delta(t-\tau-T)$, where $\mathrm{T}$ is the time constant, equation (2.12) describes the linear multiplier with fixed-time delay (2.3), where $b=0$.

In general, the linear multiplier with memory, which describes the relationship between the factor $\mathrm{X}(\mathrm{t})$ and the indicator $\mathrm{Y}(\mathrm{t})$, can described by the equation

$$
Y(t)=\int_{0}^{t} M(t, \tau) \cdot X(\tau) d \tau+b(t)
$$

where $b(t)$ is a free term that does not depend on the exogenous variable $X(t)$. Note that we can consider the memory function

$M_{b}(t, \tau)=M(t, \tau)+b(\tau) \cdot \delta(t-\tau)$, 
where $\delta(t-\tau)$ is the Dirac delta-function and $\tau \in[0, t]$. Then we have

$$
Y(t)=\int_{0}^{t} M_{b}(t, \tau) \cdot X(\tau) d \tau=\int_{0}^{t} M(t, \tau) \cdot X(\tau) d \tau+b(t) .
$$

As a result, in many cases, we can consider equation (2.12) instead of (2.13).

\subsection{Multiplier with Fading Memory}

The first mathematical description of the memory fading (dissipation) has been proposed by Volterra, V. (2005). Then the principle of memory fading was developed by various scientists. A general mathematical formulation of this principle is complicated. For applications to economic theory we will use a simplified formulation of the memory fading that is suggested by Tarasova, V. V., Tarasov, V. E. (2018a).

We can consider an exogenous variable $\mathrm{X}(\tau)$ in the form of the Dirac delta-function $\delta(\tau-T)$, i.e., $X(\tau)=m \cdot \delta(\tau-T)$, where $T>0$ is a fixed time. Using the impact $X(\tau)=m \cdot \delta(\tau-T)$, we can consider the response $Y(t)$, which is described by linear economic multiplier. In general, this response $Y(t)=m \cdot F_{0}^{t}(\delta(\tau-T))$ is not equal to zero for $t>T$. This allows us to formulate the concept of multiplier with fading memory.

Definition. The multiplier with fading memory is the generalized multiplier (2.9), which satisfies the condition

$\lim _{t \rightarrow \infty} F_{0}^{t}(\delta(\tau-T))=0$,

where $T>0$.

The linear multiplier, which is described by equation (2.12), is the multiplier with fading memory if the memory function satisfies the condition $M(t, \tau) \rightarrow 0$ at $t \rightarrow \infty$ with fixed $\tau$.

If we consider an exogenous variable $\mathrm{X}(\tau)$, which is expressed through the Dirac delta-function $\delta(\tau-T)$ in the form $X(\tau)=x(\tau) \cdot \delta(\tau-T)$. Then equation (2.12) of the linear multiplier with memory takes the form $Y(t)=M(t, T) \cdot x(T)$ for $t>T$. As a result, the behavior of the endogenous variable $\mathrm{Y}(\mathrm{t})$, which is considered as a response on the impact (perturbation) $\mathrm{X}(\tau)$, is determined by the behavior of the memory function $\mathrm{M}(\mathrm{t}, \tau)$ with fixed constant $\tau$. If the memory function tends to zero $(M(t, \tau) \rightarrow 0)$ at $t \rightarrow \infty$ with fixed $\tau$, then the economic process completely forgotten the impact, which has been subjected in the last time (see Tarasova, V. V., Tarasov, V. E. (2018a)). In this case, the effect of the multiplier with memory (2.12) is repeatable, i.e. it is reversible in a sense. In other words, the effects of multiplier with memory did not lead to irreversible changes of the economic process and economic agents, since the memory about the impact has not been preserved forever (see Tarasova, V. V., Tarasov, V. E. (2018a)).

\subsection{Multiplier with Non-Aging Memory}

The time homogeneity means that the multiplier effect is invariant under the shift $t \rightarrow t+s$. This property can be interpreted as the absence of aging in the multiplier effect. Therefore, we can talk about a generalized multiplier with non-aging memory (see Tarasova, V. V., Tarasov, V. E. (2018a)).

Definition. The multiplier with non-aging memory is the generalized multiplier (2.9), which satisfies the condition

$F_{0+s}^{t+s}(X(\tau))=F_{0}^{t}(X(\tau))$

for all variables $t>0$ and $s>0$.

Homogeneity of time means that there are no designated points in time and the description of the economic process does not depend on the initial time point. For the multiplier $(2.12)$ with the memory function $M(t, \tau)$ the homogeneity of time is represented by the condition

$M(t+s, \tau+s)=M(t, \tau)$

This condition leads (see Tarasova, V. V., Tarasov, V. E. (2018a)), to the expression $M(t, \tau)=M(t-\tau)$.

Definition. The linear multiplier with non-aging memory is the multiplier (2.12) for which the memory function satisfies the condition $M(t, \tau)=M(t-\tau)$ for all $\tau$ and $t \geq \tau$. 
Mathematically the linear multiplier with non-aging memory is represented as the Duhamel convolution of functions, $\mathrm{Y}(\mathrm{t})=\mathrm{M}(\mathrm{t}) * \mathrm{X}(\mathrm{t})$. This type of multipliers describes the economic processes that do not depend on the choice of the time origin. For these processes, we can always choose the beginning of the reference time at $\mathrm{t}=0$.

If the effect of multiplier with memory does not depend on some selected points in time and, thus, does not depend on the choice of the reference time, then it can be described by multiplier with non-aging memory, where the memory function $M(t, \tau)$ depends only on the difference $t-\tau$.

\subsection{Multiplier with Power-Law Memory}

It is known that power laws play an important role in economic processes Gabaix, X. (2009, 2016). Let us give a definition of the multiplier with fading memory, which fading has the power-law form.

Definition. The multiplier with fading memory (2.9) is called the multiplier with power-law memory $Y(t)=F_{0}^{t ; \alpha}(X(\tau))$ if there is the parameter $\alpha>0$ and a finite constant $m=m(\alpha)$ such that

$\lim _{t \rightarrow \infty} t^{1-\alpha} \cdot F_{0}^{t ; \alpha}(\delta(\tau-T))=m(\alpha)$

for fixed $T>0$.

If the multiplier is described by equation (2.12), then this definition can be rewritten in the following form.

Definition. The linear multiplier with fading memory (2.12) is called the multiplier with power-law memory if there is the parameter $\alpha>0$ and a finite constant $m(\alpha)$ such that the limit

$\lim _{t \rightarrow \infty} t^{1-\alpha} \cdot M(t, \tau)=m(\alpha)$

is finite for fixed $\tau$.

In order to have a correct definition of the economic multiplier with power-law memory, we should require the implementation of the following correspondence principle.

Correspondence principle for multiplier: If the fading parameter tends to zero $(\alpha \rightarrow 0)$, then the equation of the linear multiplier with power-law memory

$Y(t)=F_{0}^{t ; \alpha}(X(\tau))$

should takes the form of the standard multiplier without memory, $Y(t)=m \cdot X(t)$, where $m$ is the multiplier coefficient. In other words, the linear multiplier with power-law memory (2.21) should satisfy the condition

$\lim _{\alpha \rightarrow 0} F_{0}^{t ; \alpha}(X(\tau))=m \cdot X(t)$

for all $t \geq 0$, where $m=m(1)$.

In the simplest case of the power-law behavior of the memory fading, we can use the memory function $M(t, \tau)$ in the form $M(t-\tau)=c(\beta) \cdot(t-\tau)^{\beta}$, where $c(\beta)>0$ and $\beta>-1$ are constants. For simplification, we will use the parameters $\alpha=\beta+1$ and $m=c(\alpha-1) \cdot \Gamma(\alpha)$. In this case, the memory function takes the form

$M(t-\tau)=\frac{1}{\Gamma(\alpha)} \cdot \frac{m(\alpha)}{(t-\tau)^{1-\alpha}}$,

where $\alpha>0, t>\tau$ and $m(\alpha)$ is the numerical coefficient. Substituting expression (2.23) into equation (2.12), we get that the multiplier equation (2.12) can be written in the form

$Y(t)=m(\alpha) \cdot\left(I_{R L ; 0+}^{\alpha} X\right)(t)$

where $I_{R L ; 0+}^{\alpha}$ is the left-sided Riemann-Liouville fractional integral of the order $\alpha>0$ with respect to the time variable $t$. This fractional integral is defined, Samko, S. G. et. al. (1993), Podlubny, I. (1998), Kilbas, A. A., et. al. (2006) by the equation

$\left(I_{R L ; 0+}^{\alpha} X\right)(t):=\frac{1}{\Gamma(\alpha)} \cdot \int_{0}^{t} \frac{X(\tau) d \tau}{(t-\tau)^{1-\alpha}}$

where $\Gamma(\alpha)$ is the Gamma function, $0<t<T$. The function $\mathrm{X}(\mathrm{t})$ is assumed measurable on the interval $(0, T)$ such that the condition $\int_{0}^{T}|X(\tau)| d \tau<\infty$ is satisfied. The Riemann-Liouville integral (2.25) is a generalization of the standard n-th integration, Samko, S. G. et. al. (1993), Podlubny, I. (1998), Kilbas, A. A., et. al. (2006). 
Using equation (2.24), we can define a simplest multiplier with power-law memory, whose fading (damping) parameter is $\alpha \geq 0$.

Definition. The linear multiplier with simplest power-law memory, which is characterized by the fading parameter $\alpha>0$, is described by the equation

$Y(t)=m(\alpha) \cdot\left(I_{R L ; 0+}^{\alpha} X\right)(t)$,

where $I_{R L ; 0+}^{\alpha}$ is the left-sided Riemann-Liouville fractional integral (2.25) of the order $\alpha>0$ with respect to time, and $m(\alpha)$ is the coefficient of the multiplier with memory. In general, the coefficient $m(\alpha)$ is the function of the fading parameter $\alpha$.

We note that, due to strict equality for the order $\alpha>0$ of the integral (2.25), equation (2.26) cannot be considered for $\alpha=0$. Therefore, equation (2.26) does not include the standard multiplier equation, as a special case. To include the standard equation, it is necessary to extend the Riemann-Liouville integral (2.25) by the equation $\left(I_{R L ; 0}^{0} X\right)(t)=X(t)$. In this case, equation (2.26) of the generalized multiplier includes the standard equation of multiplier

We note that the Riemann-Liouville integral (2.25) for the order $\alpha=1$ gives the standard integral of the first order

$\left(I_{R L ; 0+}^{1} X\right)(t)=\int_{0}^{t} X(\tau) d \tau$.

For positive integer values $\alpha=n$, the Riemann-Liouville integral (2.25) has the form (see Samko, S. G. et. al. (1993), Kilbas, A. A., et. al. (2006)) of the standard $n$-th integral

$\left(I_{R L ; 0+}^{n} X\right)(t)=\int_{0}^{t} d t_{1} \int_{0}^{t_{1}} d t_{2} \ldots \int_{0}^{t_{n-1}} d t_{n} X\left(t_{n}\right)=\frac{1}{(n-1) !} \cdot \int_{0}^{t}(t-\tau)^{n-1} X(\tau) d \tau$.

For $\alpha=1$, multiplier equation (2.26) takes the form

$Y(t)=m(1) \cdot \int_{0}^{t} X(\tau) d \tau$

Note that differentiation of equation (2.29) with respect to the variable $t$ and the use of the standard Newton-Leibniz theorem, gives

$\frac{d}{d t} Y(t)=m(1) \cdot X(t)$

Equation (2.30) can be written in the form

$X(t)=a \cdot \frac{d}{d t} Y(t)$

which can be interpreted as the standard accelerator equation, where $a=\frac{1}{m(1)}$ is the accelerator coefficient, $\mathrm{Y}(\mathrm{t})$ is an exogenous variable and $\mathrm{X}(\mathrm{t})$ is an endogenous variable. As a result, the equation of the generalized multiplier includes the standard accelerators as a special case.

\subsection{Multiplier with Generalized Power-Law Memory}

In order to describe the economic dynamics with more complex memory, we can use the generalized fractional calculus Kiryakova, V. (1994, 2008). For example, we can define the memory function (Tarasova, V. V., Tarasov, V. E. (2018a), p. 136) by the expression

$M(t, \tau)=M_{\sigma}^{\alpha, \eta}(t, \tau):=\frac{\sigma \cdot t^{\sigma(\alpha+\eta)}}{\Gamma(\alpha)} \cdot \frac{m(\alpha) \cdot \tau^{\sigma(\eta+1)-1}}{\left(t^{\sigma}-\tau^{\sigma}\right)^{1-\alpha}}$,

where $\sigma>0, \eta$ is the real number and $\alpha>0$. Substitution of expression (2.32) into equation (2.12) gives the equation of the multiplier with generalized power-law memory (the GPLM multiplier) in the form

$Y(t)=m(\alpha) \cdot\left(I_{0+; \sigma, \eta}^{\alpha} X\right)(t)$,

where $I_{0+; \sigma, \eta}^{\alpha}$ is the left-sided Erdelyi-Kober fractional integral of the order $\alpha>0$ with respect to time variable form, Samko, S. G. et. al. (1993), Kilbas, A. A., et. al. (2006). If $\sigma=1$, then the Erdelyi-Kober fractional integral (2.33) takes the form of the Kober fractional integral (see Kilbas, A. A., et. al. (2006), p. 105-106). If $\eta=0$ and $\sigma=1$, then the Erdelyi-Kober fractional integral (2.33) is represented by the Riemann-Liouville fractional integral (2.25) such that

$\left(I_{0+; 1,0}^{\alpha} X\right)(t)=t^{-\alpha} \cdot\left(I_{R L ; 0+}^{\alpha} X\right)(t)$. 
The Erdelyi-Kober fractional integration $\left(I_{E K, a+; \sigma, \eta}^{\alpha} X\right)(t)$ is a linear map of the space $C_{\mu}$ into itself (see Theorem 2.2. of Luchko, Yu., Trujillo, J. J. (2007), p. 253) if $\mu \geq-\sigma(\eta+1)$. The space of functions $C_{\mu}$ consists of all functions $\mathrm{X}(\mathrm{t})$ with $t>0$, that can be represented in the form $X(t)=t^{p} \cdot f(t)$ with $p>\mu$ and $f(t) \in C([0, \infty)$

The economic multiplier (2.33) can be called the linear multiplier with generalized power-law memory.

The multiplier with memory (2.33) with the exogenous variable $X(\tau)=\delta(\tau-T)$ gives the expression

$Y(t)=\int_{0}^{t} M_{\sigma}^{\alpha, \eta}(t, \tau) \cdot \delta(T-\tau) d \tau=\frac{\sigma \cdot m(\alpha) \cdot T^{\sigma(\eta+1)-1}}{\Gamma(\alpha)} \cdot t^{-\sigma(\alpha+\eta)} \cdot\left(t^{\sigma}-\tau^{\sigma}\right)^{\alpha-1}$,

which means that the multiplier (2.33) is a multiplier with fading memory of power-law type.

\subsection{Multiplier with Complete (Ideal) Memory}

Let us consider a finite time interval $[0, t]$, in which the constant exogenous variable $X(t)=1$ influences on the indicator $\mathrm{Y}(\mathrm{t})$. If the endogenous variable is constant for all $t>0$, i.e. $F_{0}^{t}(1)=$ const, then the multiplier can be interpreted as a constant preserving. For the linear multiplier (2.12), this condition means that the constant exogenous variable gives the constant endogenous variable. In other words, the memory remembers unchanged variable infinitely long. For the linear multiplier (2.12), this condition leads to the following restriction on the memory function

$\int_{0}^{t} M(t, \tau) d \tau=m$

for all $t \geq 0$ and $m$ is a finite constant ( $m=$ const). Condition (2.36) means that equation (2.12) should give $Y(t)=m=$ const for $X(t)=1$ tor all $t \geq 0$.

If the memory is non-aging memory such that $M(t, \tau)=m \cdot M(t-\tau)$, then condition (2.36) has the form

$\int_{0}^{t} M(\tau) d \tau=1$

The function $\mathrm{M}(\mathrm{t})$, which satisfies the normalization condition (2.37) is often called the weighting function. This type of memory functions $\mathrm{M}(\mathrm{t})$ is often used to describe economic processes with continuously distributed lag. The existence of the time delay (lag) is connected with the fact that the processes take place with a finite speed, and the change of the economic factor does not lead to instant changes of indicator that depends on it.

If condition (2.37) holds, the economic process goes through all the states without any losses. In this case the multiplier with memory can be interpreted with the complete (perfect, ideal) memory. The multipliers of these types are used to describe processes with continuously distributed lag Allen, R. G. D. (1959), p. 25-26.

Let us consider equation (2.33) for unit exogenous variable $X(t)=1$. Using equation (2.33) with $\mathrm{X}(\mathrm{t})=1$ and equation 2.2.4.8 of Prudnikov, A. P., et. al. (1986), p. 296, in the form

$\int_{0}^{t} \tau^{\sigma(\eta+1)-1} \cdot\left(t^{\sigma}-\tau^{\sigma}\right)^{\alpha-1} d \tau=\sigma^{-1} \cdot t^{\sigma(\alpha+\eta)} \cdot B(\eta+1, \alpha)$,

where $B(x, y):=\frac{\Gamma(x) \cdot \Gamma(y)}{\Gamma(x+y)}$ is the beta function (the Euler integral of first kind), we get

$Y(t)=m(\alpha) \cdot\left(I_{0+; \sigma, \eta}^{\alpha} 1\right)(t)=\frac{m(\alpha)}{\Gamma(\alpha)} \cdot B(\eta+1, \alpha)=\frac{m(\alpha) \cdot \Gamma(\eta+1)}{\Gamma(\eta+\alpha+1)}$.

As a result, we get that the multiplier with memory, which is described by equation (2.33), can be considered as a constant preserving multiplier. The multiplier with memory, which is described by equation (2.33), allows us to describe processes that remember a constant value of exogenous variables during the infinitely long period of time. Note that the multiplier (2.33) cannot be considered as a multiplier with non-aging memory, since $M(t, \tau) \neq M(t-\tau)$ in general.

\subsection{Multipliers with Multi-Parametric Power-Law Memory}

In the equations of multipliers with power-law memory, which are discussed above, the power-law fading is characterized by one parameter $\alpha \geq 0$ only. In economic models we can take into account the presence of different types of memory fading, which characterize the different types of economic agents. In the simplest 
multi-parametric case, the multiplier with fading memory can be considered as a sum of multipliers with one-parametric memory fading. For example, we can use the equation of the linear multiplier with N-parametric power-law memory in the form

$Y(t)=\sum_{k=1}^{N} m_{k}(\alpha) \cdot\left(I_{R L ; 0+}^{\alpha_{k}} X\right)(t)$.

where $\alpha_{k}>0(k=1,2, \ldots N)$, and $m_{k}(\alpha)(k=1,2, \ldots N)$ are numerical coefficients. Using the memory with multi-parametric power-law fading, we can consider economic models that allow us to describe the memory effects in economy with different type of economic agents.

\subsection{Multiplier with Distributed Power-Law Memory}

In general, the parameter $\alpha$ of memory fading can be distributed on the interval $\left[\alpha_{1}, \alpha_{2}\right]$, where the distribution is described by a weight function $\rho(\alpha)$. The functions $\rho(\alpha)$ describes distribution of the parameter of the memory fading on a set of economic agents. This is important for the economics, since various types of economic agents may have different parameters of memory fading. In this case, we should consider the multipliers with memory function, which depends on the weight function $\rho(\alpha)$ and the interval $\left[\alpha_{1}, \alpha_{2}\right]$.

For the memory with power-law fading of distributed order, we can use the memory function

$M(t-\tau)=M_{\rho(\alpha)}^{\left[\alpha_{1}, \alpha_{2}\right]}(t-\tau)=\int_{\alpha_{1}}^{\alpha_{2}} \frac{\rho(\alpha)}{\Gamma(\alpha)} \frac{m}{(t-\tau)^{1-\alpha}} d \alpha$,

where $\alpha_{2}>\alpha_{1} \geq 0, m=m\left(\left[\alpha_{1}, \alpha_{2}\right]\right)$ and the weight function $\rho(\alpha)$ satisfies the normalization condition $\int_{\alpha_{1}}^{\alpha_{2}} \rho(\alpha) d \alpha=1$

Substituting (2.42) into equation (2.12), we obtain the integral equation of the multiplier with distributed power-law fading in the form

$Y(t)=m\left(\left[\alpha_{1}, \alpha_{2}\right]\right) \cdot\left(I_{0+}^{\left[\alpha_{1}, \alpha_{2}\right]} X\right)(t)$,

where $I_{0+}^{\left[\alpha_{1}, \alpha_{2}\right]}$ is the Riemann-Liouville fractional integral of distributed order.

Using the left-sided Riemann-Liouville integral of the order $\alpha>0$, equation of the multiplier with distributed power-law fading takes the form

$Y(t)=m\left(\left[\alpha_{1}, \alpha_{2}\right]\right) \cdot \int_{\alpha_{1}}^{\alpha_{2}} \rho(\alpha) \cdot\left(I_{0+}^{\alpha} X\right)(t) d \alpha$,

where $m\left(\left[\alpha_{1}, \alpha_{2}\right]\right)$ is the coefficient of the multiplier with distributed memory. In equation (2.44) we use the fractional integral of distributed order

$\left(I_{0+}^{\left[\alpha_{1}, \alpha_{2}\right]} X\right)(t):=\int_{\alpha_{1}}^{\alpha_{2}} \rho(\alpha) \cdot\left(I_{0+}^{\alpha} X\right)(t) d \alpha$,

where $\rho(\alpha)$ satisfies the normalization condition (2.42) and $\alpha_{2}>\alpha_{1} \geq 0$. In equation (2.45) the integration with respect to time and the integration with respect to order can be permuted for a wide class of functions $\mathrm{X}(\tau)$. As a result, equation (2.45) can be represented in the form

$\left(I_{0+}^{\left[\alpha_{1}, \alpha_{2}\right]} X\right)(t):=\int_{0}^{t} M_{\rho(\alpha)}^{\left[\alpha_{1}, \alpha_{2}\right]}(t-\tau) \cdot X(\tau) d \tau$,

where the kernel $M_{\rho(\alpha)}^{\left[\alpha_{1}, \alpha_{2}\right]}(t-\tau)$ is defined by expression (2.41). The concept of the integration and differentiation of distributed order was first proposed by Caputo, M. (1995) and then developed by Bagley, R. L., Torvik, P. J. (2000a,b), Caputo, M. (2001), Lorenzo, C. F., Hartley, T. T. (2002), Jiao, Z., et. al. (2012).

\subsection{Multiplier with Uniform Distributed and Non-Singular Distributed Memory}

In the simplest case, we can use the continuous uniform distribution (CUD) that is defined by the expression $\rho(\alpha)=\left\{\frac{1}{\alpha_{2}-\alpha_{1}} 0\right.$ for $\alpha \in\left[\alpha_{1}, \alpha_{2}\right]$ for $\alpha \in\left(-\infty, \alpha_{1}\right) \cup\left(\alpha_{2}, \infty\right)$

For the distribution function (2.47), the memory function (2.41) has the form

$M_{C U D}^{\left[\alpha_{1}, \alpha_{2}\right]}(t-\tau)=m\left(\left[\alpha_{1}, \alpha_{2}\right]\right) \cdot W\left(\alpha_{1}, \alpha_{2}, t-\tau\right)$,

we use the function 


$$
W(\alpha, \beta, t):=\frac{1}{(\beta-\alpha) \cdot t} \cdot V i(\alpha, \beta, t)=\frac{1}{(\beta-\alpha) \cdot t} \cdot \int_{\alpha}^{\beta} \frac{t^{\xi} d \xi}{\Gamma(\xi)} .
$$

The fractional integrals and derivatives of the uniform distributed order can be expressed thought the continual fractional integrals and derivatives, which have been suggested by Nakhushev, A. M. (1998, 2003). The fractional operators, which are inverse to the continual fractional integrals and derivatives, have been proposed by Pskhu, A. V. (2004, 2005).

Using the continual fractional integral, which have been suggested by Nakhushev, A. M. (1998, 2003), (see also Pskhu, A. V. (2004, 2005)), we define the fractional integral of uniform distributed order by the equation

$I_{N}^{[\alpha, \beta]} X(t)=\frac{1}{\beta-\alpha} \cdot \int_{\alpha}^{\beta} I_{R L, a+}^{\xi} X(t) d \xi=\int_{0}^{t} W(\alpha, \beta, t-\tau) \cdot X(\tau) d \tau$,

where $\beta>\alpha>0$.

As a result, we can define the multiplier with uniform distributed memory (the UDM multiplier) by the equation $Y(t)=m([\alpha, \beta]) \cdot I_{N}^{[\alpha, \beta]} X(t)$,

where $m([\alpha, \beta])$ is the multiplier coefficient, which depends on the interval $[\alpha, \beta]$ in general.

Nakhushev, A. M. (1998, 2003) also proposed the continual fractional derivatives. Using these derivatives, we can define the fractional derivatives of uniform distributed orders. The fractional integration, which is inversed to the continual fractional derivative has been proposed by Pskhu, A. V. (2004, 2005). This fractional integral can be defined by the equation

$I_{P}^{[\alpha, \beta]} X(t)=(\alpha-\beta) \cdot \int_{0}^{t} \cdot E_{\beta-\alpha}\left[(t-\tau)^{\beta-\alpha} ; \beta\right] \cdot(t-\tau)^{\beta-1} \cdot X(\tau) d \tau$,

where $\beta>\alpha>0$ and $E_{\alpha}[z ; \beta]$ is the Mittag-Leffler function that is defined by the expression

$E_{\alpha}[z ; \beta]:=\sum_{k=0}^{\infty} \frac{z^{k}}{\Gamma(\alpha k+\beta)}$.

The Pskhu fractional integrals (2.52) can be interpreted as fractional integrals with non-singular kernel. As a result, we can define the multiplier with non-singular distributed memory (the NSDM multiplier) by the equation

$Y(t)=m([\alpha, \beta]) \cdot I_{P}^{[\alpha, \beta]} X(t)$,
where $m([\alpha, \beta])$ is the multiplier coefficient.

\subsection{Principles of Superposition and Commutativity of Multipliers with Memory}

Let us describe an important property of the linear multipliers with power-law memory. This property can be presented in the form of the following superposition principle.

Principle of superposition of multipliers with memory: The multiplier effect, which is created at a given moment by several linear multipliers with power-law memory, is equivalent to the action of a linear multiplier whose memory fading is described by the sum of the fading parameters of these multipliers, and the coefficient is equal to the product of multiplier coefficients. Symbolically, this principle can be written in the form of the identity

$F_{0}^{t ; \alpha}\left(F_{0}^{t ; \beta}(X(\tau))\right)=F_{0}^{t ; \alpha+\beta}(X(\tau))$

that is satisfied for any positive fading parameters $\alpha$ and $\beta$ of the linear multipliers.

This principle also can be written in the following form. The action of two linear multipliers with power-law memory, which are described by the equations

$Y(t)=F_{0}^{t ; \alpha}\left(m_{1} \cdot X(\tau)\right)=m_{1} \cdot F_{0}^{t ; \alpha}(X(\tau))$,
$Z(t)=F_{0}^{t ; \beta}\left(m_{2} \cdot Y(\tau)\right)=m_{2} \cdot F_{0}^{t ; \beta}(Y(\tau))$,

is equivalent to the action of the following multiplier with memory

$Z(t)=m_{1} \cdot m_{2} \cdot F_{0}^{t ; \alpha+\beta}(X(\tau))$

for all $\alpha>0$ and $\beta>0$. 
For linear multipliers with memory the suggested superposition principle states the additivity of the fading parameters and the multiplicativity of the multiplier coefficients.

Example 1. For two simplified multipliers, which are described by the equations

$Y(t)=m_{1}(\alpha) \cdot\left(I_{R L ; 0+}^{\alpha} X\right)(t)$,

$Z(t)=m_{2}(\beta) \cdot\left(I_{R L ; 0+}^{\beta} Y\right)(t)$,

the superposition principle gives the equality

$Z(t)=m_{1}(\alpha) \cdot m_{2}(\beta) \cdot\left(I_{R L ; 0+}^{\alpha+\beta} X\right)(t)$

for all $\alpha>0$ and $\beta>0$. The proof of this property is based on Theorem 2.5. of Samko, S. G. et. al. (1993) p. 46, which states that the relation

$I_{R L, a+}^{\alpha} I_{R L, a+}^{\beta} X(t)=I_{R L, a+}^{\alpha+\beta} X(t)$

is valid for any $X(t) \in L_{1}(a, b)$ if $\alpha>0$ and $\beta>0$.

Example 2. If the linear multipliers are described by the Liouville fractional integrals (see Samko, S. G. et. al. (1993) p. 95 and Kilbas, A. A., et. al. (2006) p. 87-90, we can also use the superposition principle, which can be represented by the equation

$F_{-\infty}^{t ; \alpha}\left(F_{-\infty}^{t ; \beta}(X(\tau))\right)=F_{-\infty}^{t ; \alpha+\beta}(X(\tau))$

for all $\alpha>0, \beta>0$ and $X(t) \in L_{p}(-\infty,+\infty)$, where $\alpha+\beta<1 / p$ and $p \geq 1$. The proof is based on the properties of the Liouville fractional integrals that is presented by Lemma 2.19 of Kilbas, A. A., et. al. (2006), p. 89, which states that the relation

$I_{L,+}^{\alpha} I_{L,+}^{\beta} X(t)=I_{L,+}^{\alpha+\beta} X(t)$

is valid for any $\mathrm{X}(t) \in L_{p}(-\infty,+\infty)$ if $\alpha>0$ and $\beta>0, \alpha+\beta<1 / p, p \geq 1$.

Example 3. Let us consider the linear GPLM multipliers that are described by the equations

$Y(t)=m_{1}(\alpha) \cdot\left(I_{E K, a+; \sigma, \eta+\alpha}^{\alpha} X\right)(t)$,

$Z(t)=m_{2}(\beta) \cdot\left(I_{E K, a+; \sigma, \eta+\alpha}^{\beta} Y\right)(t)$,

where the memory is described through the left-sided Erdelyi-Kober fractional integrals. The superposition principle gives that the action of accelerators (2.54) and (2.65) is equivalent to the action of the GPLM multiplier

$Z(t)=m_{1}(\alpha) \cdot m_{2}(\beta) \cdot\left(I_{E K, a+; \sigma, \eta}^{\alpha+\beta} X\right)(t)$

for all $\alpha>0$ and $\beta>0$. The proof of this property is based on the left-sided Erdelyi-Kober fractional integral. Then the superposition principle follows from Lemma 2.29 of Kilbas, A. A., et. al. (2006), p. 107, which states that the relation

$\left(I_{E K, a+; \sigma, \eta}^{\alpha} I_{E K, a+; \sigma, \eta+\alpha}^{\beta} X\right)(t)=\left(I_{E K, a+; \sigma, \eta}^{\alpha+\beta} X\right)(t)$

is valid for any $X(t) \in L_{p}(a, b)$ if $\alpha>0$ and $\beta>0, p \geq 1$.

The superposition principle states that for all linear multipliers with memory, the response at a given time, which is caused by two or more multipliers with memory, is equivalent to a linear multiplier with memory, for which the memory fading is equal to the sum of the fading parameters that can be caused by each multipliers individually. This superposition principle means that the effect of the impact on the economy of the two linear multipliers does not change, when the third multiplier with memory appears.

We can generalize the superposition principle from the simplest multipliers to the multipliers with multi-parametric memory.

Principle of superposition for multipliers with multi-parametric memory: The action of two linear multipliers with multi-parametric memory is equivalent to the action of one multiplier with multi-parametric memory, whose fading parameters are described by the all possible sums of the fading parameters of these two multipliers, and the coefficient is equal to all possible products of the corresponding multiplier coefficients. The mathematical representation of this principle has the following form: The actions of the multipliers with multi-parametric memory

$Y(t)=\sum_{k=1}^{N} m_{k}\left(\alpha_{k}\right) \cdot\left(I_{R L, a^{+}}^{\alpha_{k}} X\right)(t)$, 
$Z(t)=\sum_{l=1}^{N} m_{l}\left(\alpha_{l}\right) \cdot\left(I_{R L, a+}^{\alpha_{l}} Y\right)(t)$,

are equivalent to the action of the multiplier

$Z(t)=\sum_{k, l=1}^{N} m_{k l}\left(\alpha_{k}, \alpha_{l}\right) \cdot\left(I_{R L, a+}^{\alpha_{k l}} X\right)(t)$,

where $m_{k l}\left(\alpha_{k}, \alpha_{l}\right)=m_{k}\left(\alpha_{k}\right) \cdot m_{l}\left(\alpha_{l}\right)$ and $\alpha_{k l}=\alpha_{k}+\alpha_{l}$.

As an application of the superposition principle of linear multipliers with power-law memory, it is possible to consider a generalization of the macroeconomic model with three lags (the production-income lag, the income-spending lag, the spending-production lag), which has been suggested by Allen, R. G. D. (1959), in Section 2.9 , p. 55-58. If we consider a model, where production-income memory, the income-spending memory and the spending-production memory are taken into account, we should us the superposition principle.

The consequence of the superposition principle is the principle of permutability (commutativity) of linear multipliers with power-law memory.

Principle of commutativity of multipliers with memory: The actions of two multipliers with power-law memory commutes, i.e. the property

$F_{0}^{t ; \alpha}\left(F_{0}^{t ; \beta}(X(\tau))\right)=F_{0}^{t ; \beta}\left(F_{0}^{t ; \alpha}(X(\tau))\right)$

is satisfied if $\alpha>0$ and $\beta>0$.

The principle of commutativity states the effect of the linear multiplier with power-law memory does not depend on the sequence of impact of the multipliers. Mathematically, this principle is based on the semigroup property of fractional integration. For example of the left-sided Riemann-Liouville fractional integrals, the relation $I_{R L, a+}^{\alpha} I_{R L, a+}^{\beta} X(t)=I_{R L, a+}^{\beta} I_{R L, a+}^{\alpha} X(t)$

is valid for any $X(t) \in L_{1}(a, b)$ and $\alpha>0, \beta>0$. For the left-sided Erdelyi-Kober fractional integral, we have (see Luchko, Yu., Trujillo, J. J. (2007), p. 252) the property

$\left(I_{E K, a+; \sigma, \eta}^{\alpha} I_{E K, a+; \delta, \gamma}^{\beta} X\right)(t)=\left(I_{E K, a+; \delta, \gamma}^{\beta} I_{E K, a+; \sigma, \eta}^{\alpha} X\right)(t)$,

which leads to the implementation of the commutativity principle for the multipliers with generalized power-law memory (2.33).

The consequence of this permutability (commutativity) principle is the principle of the inseparability of the action of two (or more) multipliers with memory.

Principle of inseparability of multipliers with memory: If the actions of the two multipliers with power-law memory cannot be separated, then it is impossible to separate the corresponding memory fading parameters. The mathematical form of this principle is given by the equality

$F_{0}^{t ; \alpha}(X(\tau))=F_{0}^{t ; \alpha_{1}}\left(F_{0}^{t ; \alpha_{2}}(X(\tau))\right)=F_{0}^{t ; \alpha_{3}}\left(F_{0}^{t ; \alpha_{4}}(X(\tau))\right)$,

which holds for all $\alpha_{1}>0, \alpha_{2}>0$ and $\alpha_{3}>0, \alpha_{4}>0$ such that $\alpha_{1}+\alpha_{2}=\alpha$ and $\alpha_{3}+\alpha_{4}=\alpha$.

This principle complicates econometric studies of the effects of memory in the real economy.

Inseparability means that observing only the joint action of multipliers with memory, it is impossible to determine the fading parameters of each of them.

\section{Accelerator with Memory}

\subsection{Concept of Standard Accelerator}

In addition to the concept of multiplier, the economic analysis uses the concept of accelerator Clark, J. M. (1917), Allen, R. G. D. (1959), p. 73, Allen, R. G. D. (1968), p. 81. In macroeconomics, the accelerator describes how much the change in the value of the indicator (the endogenous variable) in response of a single relative increase of the factor (the exogenous variable). 
The concept of accelerator is a mathematical expression of the acceleration principle. According to this principle, to maintain the investment at the same level is necessary to keep a constant rate of growth in the national income. The preservation of income at the same level leads to a reduction in investment to zero. The acceleration principle states a direct relationship between the rate of output of an economy and the level of net investment (see Allen, R. G. D. (1959), p. 60-64).

The concept of accelerator is based on the assumption that there is a relationship between the function, which describes the rate of change of the factor (the exogenous variable), and the function of the indicator (the endogenous variable). For macroeconomic models with continuous time, the equation of the accelerator without memory and lags can be written (see Allen, R. G. D. (1959), p. 62) as the function

$Y(t)=F\left\{\frac{d X(t)}{d t}\right\}$,

where $\frac{d X(t)}{d t}$ is the rate of the exogenous variable (for example, the income) and $\mathrm{Y}(\mathrm{t})$ is the endogenous variable (for example, the induced investment). The linear accelerator equation, which expresses the dependence of the indicator $\mathrm{Y}(\mathrm{t})$ on the rate of factor $\mathrm{X}(\mathrm{t})$, has the form

$Y(t)=a \cdot \frac{d X(t)}{d t}$,

where $a$ is the acceleration coefficient that indicates the power of acceleration (see Allen, R. G. D. (1959), p. 73). The left and right sides of equation (3.2) are written for the same time. Therefore an increase or decrease in the rate of change of the factor $\mathrm{X}(\mathrm{t})$ leads to an instantaneous change in the indicator $\mathrm{Y}(\mathrm{t})$. Obviously, this is a strong restriction of macroeconomic models. In real economic processes, a change of the rate of the factor $\mathrm{X}(\mathrm{t})$ at time $t$ cannot immediately lead to a change of the indicator $\mathrm{Y}(\mathrm{t})$.

We note that formula (3.2) can be written in the form

$\frac{d X(t)}{d t}=A \cdot Y(t)$

where $A=1 / a$. Since the left and right sides of equations (3.2) and (3.3) are written for the same time, equation (3.3) can be interpreted in the opposite direction (inverse interpretation). In equation (3.3) we can consider $\mathrm{Y}(\mathrm{t})$ as an exogenous variable (the factor) and $\mathrm{X}(\mathrm{t})$ as an endogenous variable (the indicator). In this case, equation (3.2) describes how much the change of the rate of the indicator $\mathrm{X}(\mathrm{t})$ in response of change of the factor $\mathrm{Y}(\mathrm{t})$ (the exogenous variable).

The separation of variables into exogenous and endogenous ones is based on the causal relationship: the exogenous variable is the cause (the action), and the endogenous variable is the consequence (the response). The exogenous and endogenous variables can be described as input and output. In the general case, the causal relationship assumes a finite rate of process and mutual influence of economic processes.

Let us give an example of accelerators and their equations. We will designate the national income as a function $\mathrm{Y}(\mathrm{t})$ of time $\mathrm{t}$, and the induced investment through $\mathrm{I}(\mathrm{t})$. In macroeconomic models, it is assumed that the relationship between induced investment and the growth rate of income is given by the direct proportionality (the accelerator equation) in the form

$I(t)=a \cdot \frac{d Y(t)}{d t}$,

where the coefficient $a$ is interpreted as the capital-intensity coefficient for income growth Allen, R. G. D. (1959), p. 91. The positive constant $a$ is also called the investment coefficient indicating the power of the accelerator (see Allen, R. G. D. (1959), p. 62). This coefficient shows how much the investment will increase if the current rate of change of national income increases by one, Allen, R. G. D. (1959), p. 62-63.

Equation (3.4) assumes that the investment changes instantly with a change of rate of income. Moreover, equation (3.4) assumes the absence of the memory about the previous investments and the results of these investments. Equation (3.4) means that new investments do not depend on what investments were made in the past and do not depend on the history of changes in growth rate of income.

Equation (3.4) can be written in the form

$\frac{d Y(t)}{d t}=A \cdot I(t)$

where $A=\frac{1}{a}$ is interpreted as an incremental capital-output ratio or as a marginal productivity of capital. Equation (3.5) assumes that the rate of growth of income changes instantly with the growth of investment, and does not depend on investments made at previous time points. 


\subsection{Effect of Financial Accelerator}

The concept of a financial accelerator was introduced by Bernanke, B., et. al. (1996) to explain the large fluctuations in aggregate economic activity, which arises from seemingly small shocks. It was shown that these phenomena indicate the existence of an accelerator mechanism. The mechanism of the financial accelerator is determined by the relationship between the real economy and financial markets, which is based on the need of firms for external financing to invest in business. The ability of firms to borrow significantly depends on the market value of their assets. Lenders, as a rule, require borrowers to guarantee their ability to repay, often in the form of collateralized assets. Therefore, the fall in asset prices deteriorates the balance of firms and the value of assets. As a result, the ability of firms to borrow is deteriorating, which negatively affects their investments, and this, in turn, leads to a decrease in economic activity and a further decline in asset prices. This chain reaction of changes is called a financial accelerator. This financial feedback loop (the credit cycle), which, starting with a small change in the financial markets, can lead to big changes in the economy.

Note that memory effects can weaken and strengthen the effect of the financial accelerator. For example, if economic agents remember that previous changes in economic activity (caused by small shocks), which were insignificant and short-term, then the memory effect may lead to a more rapid decay in the fluctuation of economic activity. On the other hand, if agents remember that previous fluctuations, which are generated by minor shocks, were large, prolonged, and significantly worsened the economic situation, then repetition of even smaller shocks could lead to more significant changes in the economy than it had previously.

\subsection{Generalized Accelerator with Memory}

To generalize the concept of the economic accelerator to the case of processes with memory, we should take into account that the endogenous variable $\mathrm{Y}(\mathrm{t})$ at time $\mathrm{t}$ depends on the changes of the exogenous variable $X(\tau)$ and its integer derivatives $X^{(k)}(\tau) \quad(k=1,2, \ldots, n)$ on a finite time interval $0 \leq \tau \leq t$. In economic processes, the memory effects are caused with the fact that economic agents remember the history of previous changes of the exogenous variable $\mathrm{X}(\mathrm{t})$ and the influence on the changes of the endogenous variable $\mathrm{Y}(\mathrm{t})$. In the economic process with memory there exists at least one endogenous variable $\mathrm{Y}(\mathrm{t})$ at the time $\mathrm{t}$, which depends on the history of the change of $\mathrm{X}(\tau)$ and its integer derivatives $X^{(k)}(\tau) \quad(k=1,2, \ldots, n)$ at $\tau \in(0, t)$. For this reason, it is impossible to describe the processes with memory by standard accelerator equation (3.1) with the first derivative $X^{(1)}(t)$. As a result, we propose the following definition of the generalized accelerator with memory.

Definition. The generalized accelerator with memory

$Y(t)=E_{0}^{t}(X(\tau))$

is the dependence of an endogenous variable $Y(t)$ at the time $t \geq 0$ on the histories of the changes of the exogenous variable $X(\tau)$ and their derivatives of integer orders up to the order $n$ on a finite time interval $0 \leq \tau \leq t$. In a symbolic form, we write this in the form of the expression

$Y(t)=F_{0}^{t}\left(X^{(n)}(\tau)\right)$

where $X^{(n)}(\tau)=\frac{d^{n} X(\tau)}{d \tau^{n}}$ is the derivative of $X(\tau)$ of the integer positive order $n$ with respect to time $\tau \in[0, t]$, and $F_{0}^{t}$ is an operator that transforms each history of changes of $X(\tau)$ and $X^{(k)}(\tau), k=1, \ldots, n$ for $\tau \in[0, t]$ into the appropriate history of changes of $Y(\tau)$ with $\tau \in[0, t]$.

Equation (3.7) can be interpreted as a multiplier equation, in which the exogenous variable is the derivatives $X^{(k)}(\tau)$ of integer orders $k=1, \ldots, n$ and endogenous variable is $\mathrm{Y}(\mathrm{t})$. The multiplier with memory can be considered as a special case of the suggested concept of the accelerator with memory if we include the case $X^{(0)}(\tau)=X(\tau)$ in equation (3.7)

The generalized accelerator with memory (3.6) is called linear, if the condition

$E_{0}^{t}\left(a \cdot X_{1}(\tau)+b \cdot X_{2}(\tau)\right)=a \cdot E_{0}^{t}\left(X_{1}(\tau)\right)+b \cdot E_{0}^{t}\left(X_{2}(\tau)\right)$

holds for all $\mathrm{a}$ and $\mathrm{b}$ from the field of scalars (for example, $\mathrm{a}$ and $\mathrm{b}$ are real numbers). Using (3.6) and the property

$\left(a \cdot X_{1}(\tau)+b \cdot X_{2}(\tau)\right)^{(n)}=a \cdot X_{1}^{(n)}(\tau)+b \cdot X_{2}^{(n)}(\tau)$,

equality (3.8) gives the condition

$F_{0}^{t}\left(a \cdot X_{1}^{(n)}(\tau)+b \cdot X_{2}^{(n)}(\tau)\right)=a \cdot F_{0}^{t}\left(X_{1}^{(n)}(\tau)\right)+b \cdot F_{0}^{t}\left(X_{2}^{(n)}(\tau)\right)$ 
which is holds for all real numbers a and $b$.

We can consider the linear operator $F_{0}^{t}$ in the form of the Volterra integral operator with the memory function $M(t, \tau)$. It allows us to define the linear generalized accelerator with memory of the special kind.

Definition. The generalized accelerator with memory (3.7) is called the linear generalized accelerator with memory function $M(t, \tau)$ if the accelerator (3.7) can be represented in the form

$Y(t)=\int_{0}^{t} M(t, \tau) \cdot X^{(n)}(\tau) d \tau$,

where $n$ is positive integer number and the function $M(t, \tau)$ describes the dynamic memory.

We assume that expression (3.11) has a mathematical sense, i.e., we assume that the integral (3.11) exists. Some properties and examples of the accelerators with memory are considered in this paper below. If the function $M(t, \tau)$ is expressed in terms of the Dirac delta-function $(M(t, \tau)=a \cdot \delta(t-\tau)$, then equation (3.11) with $\mathrm{n}=1$ takes the form of the standard accelerator equation $Y(t)=a \cdot X^{(1)}(t)$.

We can see that the generalized accelerator with memory, which connects the endogenous variable $\mathrm{Y}(\mathrm{t})$ and the exogenous variable $\mathrm{X}(\tau)$ with $\tau \in[0, t]$, can be mathematically interpreted as a generalized multiplier with memory, which connects the endogenous variable $\mathrm{Y}(\mathrm{t})$ and the derivative $X^{(n)}(\tau)$ of the exogenous variable $\mathrm{X}(\tau)$ with $\tau \in[0, t]$.

Note that the linear generalized accelerator with memory, which is characterized by the memory function $M(t, \tau)$, can also be defined by the equations

$$
\begin{aligned}
& Y(t)=\frac{d^{n}}{d t^{n}} F_{0}^{t}(X(\tau))=\frac{d^{n}}{d t^{n}} \int_{0}^{t} M(t, \tau) \cdot X(\tau) d \tau, \\
& Y(t)=\frac{d^{n-k}}{d t^{n-k}} F_{0}^{t}\left(X^{(k)}(\tau)\right)=\frac{d^{n-k}}{d t^{n-k}} \int_{0}^{t} M(t, \tau) \cdot X^{(k)}(\tau) d \tau,
\end{aligned}
$$

where $n \geq k$. The generalized accelerator with memory can also be described by the equation

$$
Y(t)=D_{0+}^{\alpha-\beta} \int_{0}^{t} M(t, \tau) \cdot\left(D_{0+}^{\beta} X\right)(\tau) d \tau,
$$

where $D_{0+}^{\alpha-\beta}$ and $D_{0+}^{\beta}$ with $\alpha \geq \beta$ are derivatives of fractional (non-integer) orders (see Samko, S. G. et. al. (1993) and Kilbas, A. A., et. al. (2006)).

Let us consider an exogenous variable $\mathrm{X}(\tau)$ that is represented through the Dirac delta-function $\delta(\tau-T)$, i.e., $X(\tau)=a \cdot \delta(\tau-T)$, where $T>0$ is a fixed time. For this impact, we consider properties of the response $Y(t)$, which is described by the linear economic accelerator with memory $Y(t)=a \cdot F_{0}^{t}\left(X^{(n)}(\tau)\right)$ for $t>T$. We can give the following definition.

Definition. The generalized accelerator (3.7) is called the accelerator with fading memory if the condition $\lim _{t \rightarrow \infty} F_{0}^{t}\left(\delta^{(n)}(\tau-T)\right)=0$

is satisfied for $T>0$.

Let us consider the linear accelerator, which is described by equation (3.11) with the exogenous variable $X(\tau)=\delta(\tau-T)$. The substitution of $X(\tau)=\delta(\tau-T)$ into equation (3.11) with integer $n \geq 1$ gives

$$
Y(t)=\int_{0}^{t} M(t, \tau) \cdot \delta^{(n)}(\tau-T) d \tau
$$

Using the properties of the delta-function, we get the response $\mathrm{Y}(\mathrm{t})$ for $t>T$ in the form

$$
Y(t)=(-1)^{n} \cdot\left(\frac{\partial^{n} M(t, \tau)}{\partial \tau^{n}}\right)_{\tau=T} \text {. }
$$

As a result, accelerator (3.11) is the accelerator with fading memory if the endogenous variable tends to zero ( $Y(t) \rightarrow 0)$ at $t \rightarrow \infty$. 
If we consider equation (3.16) with $M(t, \tau)=M(t-\tau)$, where the factor $\mathrm{X}(\tau)$ is represented by the Dirac delta-function $\delta(\tau-T)$. Substitution of $X(\tau)=\delta(\tau-T)$ into equation (3.11) gives the response $\mathrm{Y}(\mathrm{t})$ for $t>T$ in the form

$Y(t)=\frac{\partial^{n} M(t, T)}{\partial t^{n}}$

where we use $\frac{\partial M(t, \tau)}{\partial t}=-\frac{\partial M(t, \tau)}{\partial \tau}$.

Note that the concept of the accelerator with memory for the discrete time approach has been suggested by Tarasova, V. V., Tarasov, V. E. (2016a).

\subsection{Accelerator with Power-Law Memory}

Let us give a definition of the accelerators with memory, which fading has the power-law form.

Definition. The accelerator with fading memory $Y(t)=F_{0}^{t}\left(X^{(n)}(\tau)\right)$ is called the accelerator with power-law memory if there is a parameter $\alpha>0$ and a finite non-zero constant a such that the limit

$\lim _{t \rightarrow \infty}(t-T)^{\alpha+1} \cdot F_{0}^{t}\left(\delta^{(n)}(\tau-T)\right)=a(\alpha)$

exists for fixed $T>0$. In this case, we will use the notation $F_{0}^{t}\left(X^{(n)}(\tau)\right)=F_{0}^{t ; \alpha}\left(X^{(n)}(\tau)\right)$.

The linear accelerator (3.11) with fading memory

$Y(t)=F_{0}^{t}\left(X^{(n)}(\tau)\right)=\int_{0}^{t} M(t, \tau) \cdot X^{(n)}(\tau) d \tau$

is called the accelerator with power-law memory if there is a parameter $\alpha>0$ such that the limit

$\lim _{t \rightarrow \infty} t^{n-(\alpha+1)} \cdot M(t, \tau)=a(\alpha)$

is finite for fixed $\tau>0$.

In this definition, we can take into account equations (3.16) and (3.17). It allows us to replace the equation (3.21) by the finiteness condition of the limit

$\lim _{t \rightarrow \infty} t^{-(\alpha+1)} \cdot \frac{\partial^{n} M(t, \tau)}{\partial \tau^{n}}=a(\alpha)$

for fixed values $\tau>0$.

In order to have a correct definition of the economic accelerator, we should require the implementation of the following correspondence principle.

Correspondence principle for accelerator: If the fading parameter $\alpha \geq 0$ tends to unity ( $\alpha \rightarrow 1)$, then equation (3.20) of the linear accelerator with power-law memory should takes the form of the standard accelerator without memory, $Y(t)=a(1) \cdot X^{(1)}(t)$, where $a(1)$ is the accelerator coefficient. In other words, the linear accelerator with power-law memory (3.20) should satisfy the condition

$\lim _{\alpha \rightarrow 1} F_{0}^{t ; \alpha}\left(X^{(n)}(\tau)\right)=a(1) \cdot X^{(1)}(t)$

for all $t \geq 0$, when the parameter $n$ is equal to unity $(n=1)$.

It is obvious that the equation $Y(t)=F_{0}^{t}\left(X^{(n)}(\tau)\right)$, which describes a generalized accelerator with memory, should give the equation of the generalized multiplier with memory $Y(t)=F_{0}^{t}(X(\tau))$, when we use $n=0$ and $X^{(0)}(\tau)=X(\tau)$ for all $\tau \geq 0$.

\subsection{Examples of Accelerators with Memory}

\subsubsection{Accelerator with Simplest Power-Law Memory}

In the simplest case of the power-law behavior of the memory fading, we can use the memory function $M(t, \tau)$ in the form $M(t-\tau)=c(\beta) \cdot(t-\tau)^{\beta}$, where $c(\beta)>0$ and $\beta>-1$ are constants. For simplification, we will use the parameters $\alpha=n-\beta-1$ and $c(\beta)=c(n-\alpha-1)=\frac{a(\alpha)}{\Gamma(n-\alpha)}$,i.e., $a(\alpha)=c(n-\alpha-1) \cdot \Gamma(n-\alpha)$. In this case, the memory function takes the form

$M(t, \tau)=M_{n-\alpha}(t-\tau)=\frac{1}{\Gamma(n-\alpha)} \cdot \frac{a(\alpha)}{(t-\tau)^{\alpha-n+1}}$,

where $n:=[\alpha]+1, \alpha>0, t>\tau$ and $a=a(\alpha)$ is the numerical coefficient. Substitution of expression (3.23) into equation (3.11) gives the accelerator equation (3.11) in the form 
$Y(t)=a(\alpha) \cdot\left(D_{C ; 0+}^{\alpha} X\right)(t)$,

where $D_{0+}^{\alpha}$ is the left-sided Caputo fractional derivative of the order $\alpha \geq 0$ of the function $\mathrm{X}(\mathrm{t})$ that is defined (see Kilbas, A .A., (2006), p. 92) by the equation

$$
\left(D_{C ; 0+}^{\alpha} X\right)(t)=\left(I_{R L ; 0+}^{n-\alpha} X^{(n)}\right)(t)=\frac{1}{\Gamma(n-\alpha)} \cdot \int_{0}^{t} \frac{X^{(n)}(\tau) d \tau}{(t-\tau)^{\alpha-n+1}},
$$

where $\Gamma(\alpha)$ is the Gamma function, $0<t<T$, and $X^{(n)}(\tau)$ is the derivative of the integer order $n:=[\alpha]+1$ (and $n=\alpha$ for integer values of $\alpha$ ) of the function $\mathrm{X}(\tau)$ with respect to the time variable $\tau$, and $I_{R L ; 0+}^{\alpha}$ is the left-sided Riemann-Liouville fractional integral of the order $\alpha>0$, Kilbas, A .A., (2006), p. 69-70. In equation (3.25), it is assumed that the function $\mathrm{X}(\mathrm{t})$ has derivatives up to (n-1)th order, which are absolutely continuous functions on the interval $[0, T]$. Equation (3.24) describes the equation of economic accelerator with memory with the power-law fading of the order $\alpha \geq 0$, where $a=a(\alpha)$ is a positive constant indicating the power of this accelerator. As a result, we obtain the following definition.

Definition. The equation of linear accelerator with memory, which is characterized by the fading parameter $\alpha \geq 0$, has the form

$$
Y(t)=a(\alpha) \cdot\left(D_{C ; 0+}^{\alpha} X\right)(t) \text {, }
$$

where $D_{C ; 0+}^{\alpha}$ is the Caputo fractional derivative (3.25) of the order $\alpha \geq 0$ with respect to time, and $a(\alpha)$ is the coefficient of the accelerator with memory.

Note that for the integer orders $\alpha=n$, the Caputo derivative coincides (see Podlubny, I. (1998), p. 79; Kilbas, A .A., (2006), p. 92-93) with the standard integer-order derivatives

$\left(D_{C ; 0+}^{n} X\right)(t)=X^{(n)}(t)$,

where we have $\left.D_{C ; 0+}^{0} X\right)(x)=X^{(0)}(t)=X(t)$ for $n=0$. This property shows that the Caputo fractional derivative is a generalization of the derivatives of the integer orders. The Caputo derivatives include the integer derivatives, as a special case.

The property of the Caputo fractional derivative allows us to consider the linear accelerator with memory, which is described by equation (3.26), as a generalization of the standard equations of accelerator and standard multiplier. These standard concepts are included into suggested concept as a special case.

Substitution of $X(\tau)=\delta(\tau-T)$ into equation (3.26) gives the response $\mathrm{Y}(\mathrm{t})$ for $t>T$ in the form

$$
Y(t)=\frac{\partial^{n} M_{n-\alpha}(t-T)}{\partial t^{n}}=\frac{a(\alpha)}{\Gamma(-\alpha)} \cdot(t-T)^{-\alpha-1}
$$

for non-integer values of order $\alpha: n-1<\alpha \leq n$. As a result, we can see that the behavior of the endogenous variable $\mathrm{Y}(\mathrm{t})$, which is considered as a response on the impact $\mathrm{X}(\tau)$, has the power-law type, where $\beta=-\alpha-1$.

As an example of the simplest linear accelerator with memory that is represented in the form

$$
Y(t)=\frac{d^{n}}{d t^{n}} F_{0}^{t}(X(\tau))=\frac{d^{n}}{d t^{n}} \int_{0}^{t} M(t, \tau) \cdot X(\tau) d \tau
$$

we can consider the equation

$$
Y(t)=a(\alpha) \cdot\left(D_{R L ; 0+}^{\alpha} X\right)(t),
$$

where $D_{R L ; 0+}^{\alpha}$ is the left-sided Riemann-Liouville fractional derivative of order $\alpha \geq 0$ of the function $X(t)$, which is defined (Kilbas, A .A., (2006), p. 92) by the equation

$$
\left(D_{R L ; 0+}^{\alpha} X\right)(t)=\frac{d^{n}}{d t^{n}}\left(I_{R L ; 0+}^{n-\alpha} X\right)(t):=\frac{1}{\Gamma(n-\alpha)} \cdot \frac{d^{n}}{d t^{n}} \int_{0}^{t} \frac{X(\tau) d \tau}{(t-\tau)^{\alpha-n+1}},
$$

where $\Gamma(\alpha)$ is the Gamma function, $0<t<T$, and $n:=[\alpha]+1$. A sufficient condition of the existence of fractional derivatives (3.31) is $X(t) \in A C^{n-1}[0, T]$ (see Samko, S. G. et. al. (1993) p. 36-37). The space $A C^{n}[0, T]$ consists of functions $\mathrm{X}(\mathrm{t})$, which have continuous derivatives up to order $\mathrm{n}-1$ on $[0, T]$ with absolutely continuous functions $X^{(n-1)}(t)$ on the interval $[0, T]$.

We note that the Riemann-Liouville derivatives of orders $\alpha=1$ and $\alpha=0$ give the expressions $\left(D_{R L ; 0+}^{1} X\right)(t)=X^{(1)}(t)$ and $\left(D_{R L ; 0+}^{0} X\right)(t)=X(t)$, respectively (see Kilbas, A. A., et. al. (2006) p. 70).

Note that the function 
$X(t)=\sum_{k=1}^{n} x_{k} \cdot t^{\alpha-k}$

where $x_{k}$ are arbitrary constants and $n:=[\alpha]+1$, plays the same role (see Samko, S. G. et. al. (1993), p. 36-37 and Kilbas, A. A., et. al. (2006), p. 72] for the Riemann-Liouville derivative of orders $\alpha$ as a constant function for the first derivative and as the functions

$X(t)=\sum_{k=1}^{n} c_{k} \cdot t^{k-1}=\sum_{k=1}^{n} x_{k} \cdot t^{n+1-k}$

for the derivative of positive integer order $n$.

For positive integer values $\alpha=n$ equation (3.26) takes the form

$Y(t)=a(n) \cdot \frac{d^{n} X(t)}{d t^{n}}$.

As a result, the concept of an accelerator with memory of the order $\alpha \geq 0$ includes, as special cases, the equations of "higher-order accelerators" (3.34), which are usually called the linear dynamic elements of order $n$. For $\alpha=n=1$, equations (3.26) and (3.30) coincide with equation (3.2), that is, (3.26) and (3.30) include the standard accelerator equation, as a special case.

Let us note that the proposed concept of the accelerator with memory includes not only the standard concept of the accelerator as special case, but it also includes the standard concept of the multiplier.

The simplest linear accelerator with memory, which is described by equation (3.26), gives the equations of the standard accelerator and multiplier for the values $\alpha=0$ and $\alpha=1$. For the cases $\alpha=0$ and $\alpha=1$, we should use the properties of the Caputo derivative $\left(D_{0+}^{1} X\right)(t)=X^{(1)}(t)$, and $\left(D_{C ; 0+}^{0} X\right)(t)=X(t)$, (see Podlubny, I. (1998), p. 79; Kilbas, A .A., (2006), p. 92-93). For $\alpha=0$ we use $\left(D_{C ; 0+}^{0} X\right)(t)=X(t)$, and equation (3.26) gives $Y(t)=a(0) \cdot X(t)$,

which is the standard equation of multiplier without memory, where the multiplier coefficient is equal to the accelerator coefficient, i.e. $m=a(1)$. For $\alpha=1$, we use $\left(D_{C ; 0+}^{1} X\right)(t)=X^{(1)}(t)$. Then equation $(3.26)$ takes the form

$$
Y(t)=a(1) \cdot \frac{d X(t)}{d t},
$$

which is the standard equation of the accelerator without memory.

As a result, equation (3.26) and (3.30) can be considered as a generalization of standard accelerator equation (3.2) and multiplier equation (3.35). Equations of the standard accelerator and multiplier are included in equations (3.26) and (3.30) as special cases. In addition to this inclusion, the suggested concept of the accelerator with memory includes the wide spectrum of intermediate dependences of the indicator on the factor. Equations (3.26) and (3.30) allow us to take into account the fact that the economic process depends on the history of previous changes of factors and indicators on the finite time interval.

\subsubsection{Accelerator with Generalized Power-Law Memory}

In order to describe the economic dynamics with more complex memory, we can use the generalized fractional calculus, Kiryakova, V. (1994, 2008). For example, we can consider the accelerator with generalized power-law memory in the form

$$
Y(t)=F_{0}^{t}\left(\prod_{k=1}^{n}\left(p \cdot \frac{d}{d \tau}+q\right) X(\tau)\right)
$$

where $q, p$ are some numbers and $n-1<\alpha \leq n$, instead of the equation $Y(t)=F_{0}^{t}\left(X^{(n)}(\tau)\right)$.

Example 1. For the linear accelerator we can use the memory function

$M(t, \tau)=M_{\sigma}^{n-\alpha, \eta+\alpha}(t, \tau)=\frac{\sigma \cdot t^{-\sigma(n+\eta)}}{\Gamma(n-\alpha)} \cdot \frac{a(\alpha) \cdot \tau^{\sigma(\eta+\alpha+1)-1}}{\left(t^{\sigma}-\tau^{\sigma}\right)^{\alpha-n+1}}$.

As a result, the linear accelerator with generalized power-law memory (the GPLM accelerator) is defined by the equation

$$
Y(t)=a(\alpha) \cdot\left(D_{E K C ; a+; \sigma, \eta}^{\alpha} X\right)(t),
$$

where $D_{E K C ; 0+; \sigma, \eta}^{\alpha}$ is the Caputo modification of the Erdelyi-Kober fractional derivative (see Luchko, Yu., Trujillo, J. J. (2007), p. 260) of the order $\alpha>0$ with respect to time variable. The Caputo modification of the Erdelyi-Kober fractional derivative has been suggested by Luchko, Yu., Trujillo, J. J. (2007), p. 260, and then it 
has been generalized by Kiryakova, V., Luchko, Yu. (2013). The Caputo modification of the Erdelyi-Kober fractional derivative is defined by Luchko, Yu., Trujillo, J. J. (2007), p. 260, in the form

$$
\left(D_{E K C, a+; \sigma, \eta}^{\alpha} X\right)(t):=\frac{\sigma \cdot t^{\sigma(n+\eta)}}{\Gamma(n-\alpha)} \int_{a}^{t} \frac{\tau^{\sigma(\eta+\alpha+1)-1}}{\left(t^{\sigma}-\tau^{\sigma}\right)^{\alpha-n+1}} \prod_{k=1}^{n}\left(\frac{\tau}{\sigma} \cdot \frac{d}{d \tau}+\eta+k\right) X(\tau) d \tau .
$$

For $\eta=0, \sigma=1$, the accelerator equation (3.40) gives the equations of accelerator with simplest power-law memory, which is expressed through the Caputo fractional derivative.

Example 2. We can also use the left-sided Erdelyi-Kober fractional derivatives of the order $\alpha>0$ Kilbas, A. A., et. al. (2006), p. 108 that are defined by the equation

$$
\left(D_{E K, a+; \sigma, \eta}^{\alpha} X\right)(t):=t^{-\sigma \eta}\left(\frac{1}{\sigma \cdot t^{\sigma-1}} \cdot \frac{d}{d t}\right)^{n} t^{\sigma(n+\eta)}\left(I_{E K, a+; \sigma, \eta+\alpha}^{n-\alpha} X\right)(t) \text {, }
$$

where $n-1<\alpha \leq n$. Here $I_{E K, a+; \sigma, \eta}^{\alpha}$ is the left-sided Erdelyi-Kober fractional integral of the order $\alpha>0$ with respect to time variable Kilbas, A .A., et. al. (2006). p. 105; Samko, S. G., et. al. (1993), p. 322-325. (see also Luchko, Yu., Trujillo, J. J. (2007), p. 251, where $\delta=\alpha, \beta=\sigma, \gamma=\eta$ ), which is defined by the equation

$\left(I_{E K, a+; \sigma, \eta}^{\alpha} X\right)(t):=\frac{\sigma \cdot t^{-\sigma(\alpha+\eta)}}{\Gamma(\alpha)} \int_{a}^{t} \frac{\tau^{\sigma(\eta+1)-1}}{\left(t^{\sigma}-\tau^{\sigma}\right)^{1-\alpha}} X(\tau) d \tau$,

where $\sigma>0, \eta$ is the real number and $\alpha>0$. For $\sigma=1$, equation (3.42) takes the form of the Kober fractional integral. For $\eta=0$ and $\sigma=1$, equation (3.42) can be expressed through the Riemann-Liouville fractional integral by the equation

$\left(I_{0+; 1,0}^{\alpha} X\right)(t)=t^{-\alpha} \cdot\left(I_{0+}^{\alpha} X\right)(t)$.

Note that the Erdelyi-Kober fractional integration $\left(I_{E K, a+; \sigma, \eta}^{\alpha} X\right)(t)$ is a linear map of the space $C_{\mu}$ into itself (see Theorem 2.2. of Luchko, Yu., Trujillo, J. J. (2007), p. 253.) if $\mu \geq-\sigma(\eta+1)$. The function space $C_{\mu}$ consists of all functions $\mathrm{X}(\mathrm{t}), t>0$, that can be represented in the form $X(t)=t^{p} \cdot f(t)$ with $p>\mu$ and $f(t) \in C([0, \infty)$.

Another type of accelerator with generalized power-law memory (the GPLM accelerator) can be defined by the equation

$Y(t)=a(\alpha) \cdot\left(D_{E K, a+; \sigma, \eta}^{\alpha} X\right)(t)$,

where $D_{0+; \sigma, \eta}^{\alpha}$ is the left-sided Erdelyi-Kober fractional derivative of the Riemann-Liouville type of the order $\alpha>0$ with respect to time variable. In the book of Samko, S. G., et. al. (1993), p. 322, the left-sided Erdelyi-Kober fractional derivative of the Riemann-Liouville type is defined by the equation

$\left(D_{E K ; a+; \sigma, \eta}^{\alpha} X\right)(t):=t^{-\sigma(\eta-\alpha)}\left(\frac{1}{\sigma \cdot t^{\sigma-1}} \cdot \frac{d}{d t}\right)^{n} t^{\sigma(n+\eta-\alpha)}\left(I_{E K, a+; \sigma, \eta}^{n-\alpha} X\right)(t)$,

where $n-1<\alpha \leq n$. In the paper of Luchko, Yu., Trujillo, J. J. (2007), p. 254, the Erdelyi-Kober fractional derivative of the Riemann-Liouville type is defined as

$\left(D_{E K C ; a+; \sigma, \eta}^{\alpha} X\right)(t):=\prod_{k=1}^{n}\left(\frac{\tau}{\sigma} \cdot \frac{d}{d \tau}+\eta+k\right)\left(I_{E K, a+; \sigma, \eta+\alpha}^{n-\alpha} X\right)(t)$,

where $n-1<\alpha \leq n$.

\subsubsection{Accelerator with Multi-Parametric Power-Law Memory}

In the simplest linear accelerator with power-law memory, the power-law fading is characterized by one parameter $\alpha$ only. In general, the different types of economic agents can be characterized by different types of memory fading. In this case, we can use the memory function $M(t, \tau)$ as a sum of functions (3.23) with different parameters of fading memory. For example, to take into account $\mathrm{N}$ parameters of memory fading, we can use the equation

$$
Y(t)=\sum_{k=1}^{N} a_{k}\left(\alpha_{k}\right) \cdot\left(D_{C ; 0+}^{\alpha_{k}} X\right)(t)
$$

where $a_{k}\left(\alpha_{k}\right)$ are numerical coefficients. The use of the accelerators with multi-parametric power-law memory allows us to describe the memory effects in economics with different type of economic agents.

\subsubsection{Accelerator with Variable Power-Law Memory}

In some economic processes with memory, the parameter $\alpha$ of memory fading can be changed during the time, i.e. $\alpha=\alpha(t)$. The accelerator with variable power-law memory can be described by the equation $Y(t)=a(\alpha) \cdot\left(D_{0+}^{\alpha(t)} X\right)(t)$, 
where $D_{0+}^{\alpha(t)}$ is the left-sided Caputo fractional derivative of the variable order $\alpha(t) \geq 0$. For $\alpha(t)=\alpha$ equation (3.48) gives (3.26).

\subsubsection{Accelerator with Uniform Distributed and Non-Singular Distributed Memory}

In general, the parameter $\alpha$ of memory fading can be distributed on the interval $\left[\alpha_{1}, \alpha_{2}\right]$, where the distribution is described by a weight function $\rho(\alpha)$. These functions describe distributions of the parameter of memory fading on the set of economic agents. This is important for the economics, since various types of economic agents may have different parameters of memory fading. In this case, we should consider the accelerators with memory, which depend on the weight function $\rho(\alpha)$ and the interval $\left[\alpha_{1}, \alpha_{2}\right]$. Let us consider an example of the memory with power-law fading of distributed order. In the simplest case, we can use the continuous uniform distribution (CUD) that is defined by the expression

$\rho(\alpha)=\frac{1}{\alpha_{2}-\alpha_{1}}$

for the case $\alpha \in\left[\alpha_{1}, \alpha_{2}\right]$ with $\alpha_{2}-\alpha_{1}>0$, and $\rho(\alpha)=0$ for other cases.

The fractional integrals and derivatives of the uniform distributed order can be expressed thought the continual fractional integrals and derivatives, which have been suggested by Nakhushev, A. M. (1998, 2003). The fractional operators, which are inversed to the continual fractional integrals and derivatives, have been proposed by Pskhu, A. V. (2004, 2005). Using the continual fractional integrals and derivatives, which have been suggested by Nakhushev, A. M. (1998, 2003), we will define the integral and derivatives of uniform distributed order. These operators will be called the Nakhushev fractional integrals and derivatives. The corresponding inverse operators Pskhu, A. V. (2004, 2005) will be called the Pskhu fractional integrals and derivatives.

The continual fractional integrals and derivatives have been suggested by Nakhushev, A. M. (1998, 2003), (see also Pskhu, A. V. (2004, 2005)). These operators are defined by the equation

$D_{0+}^{[\alpha, \beta]} X(t):=\int_{\alpha}^{\beta} D_{0+}^{\xi} X(t) d \xi$

where $\alpha<\beta, D_{0+}^{\xi}=D_{R L, 0+}^{\xi}$ for $\xi>0$ and $D_{0+}^{\xi}=I_{R L, 0+}^{-\xi}$ for $\xi<0$. The fractional operators, which are inverse to operators $D_{0+}^{[\alpha, \beta]}$, are proposed by Pskhu, A. V. $(2004,2005)$. These operators are defined (see equation 5.1 .3 of Pskhu, A. V. (2005)) by the equations

$D_{0+}^{-[\alpha, \beta]} X(t):=\left\{-t^{\beta-1} E_{\beta-\alpha}\left[t^{\beta-\alpha} ; \beta\right] * X(t)\right.$ for $\beta>0, D^{n} D_{0+}^{-[\alpha+n, \beta+n]} X(t)$ for $-n<\beta \leq-n+1$,

where $E_{\alpha}[z ; \beta]$ is the Mittag-Leffler function that is defined by the expression

$E_{\alpha}[z ; \beta]:=\sum_{k=0}^{\infty} \frac{z^{k}}{\Gamma(\alpha k+\beta)}$.

The symbol $*$ denotes the convolution

$(f * g)(t):=\int_{0}^{t} f(\tau) \cdot g(t-\tau) d \tau=\int_{0}^{t} f(t-\tau) \cdot g(\tau) d \tau$.

In works of Pskhu, A. V. $(2004,2005)$ the notations $D_{0+}^{[\alpha, \beta]}$ and $D_{0+}^{-[\alpha, \beta]}$ are used for positive $(0<\alpha<\beta)$ and negative $(\alpha<\beta \leq 0)$ values of $\alpha$ and $\beta$. In our opinion, this leads to confusion and misunderstanding in applications. Therefore we will use new notations, which allow us to see explicitly the integration and differentiation of the fractional orders.

We will define fractional integrals and derivatives of the uniform distributed orders by the equations

$$
\begin{aligned}
& D_{N}^{[\alpha, \beta]} X(t):=\frac{1}{\beta-\alpha} \cdot D_{0+}^{[\alpha, \beta]} X(t)=\frac{1}{\beta-\alpha} \cdot \int_{\alpha}^{\beta} D_{R L, 0+}^{\xi} X(t) d \xi, \\
& I_{N}^{[\alpha, \beta]} X(t):=\frac{1}{\beta-\alpha} \cdot D_{0+}^{[-\beta,-\alpha]}=\frac{1}{\beta-\alpha} \cdot \int_{\alpha}^{\beta} I_{R L, 0+}^{\xi} X(t) d \xi,
\end{aligned}
$$

where $\beta>\alpha>0$. Equations (3.54) and (3.55) express the suggested derivatives and integrals through the operators (3.50) and (3.51). The derivative (3.54) and integral (3.55) will be called the Nakhushev fractional derivative and integral, respectively. 
Note that the Nakhushev fractional derivatives cannot be considered as inverse operators for the Nakhushev fractional integrals. Operators, which are inverse to the Nakhushev fractional derivatives and integrals, are expressed through (3.50) and (3.51) by equations

$D_{P}^{[\alpha, \beta]} X(t):=(\beta-\alpha) \cdot D_{0+}^{-[-\beta,-\alpha]} X(t)$,

$I_{P}^{[\alpha, \beta]} X(t):=(\beta-\alpha) \cdot D_{0+}^{-[\alpha, \beta]}$,

where $\beta>\alpha>0$. These integrals and derivatives will be called the Pskhu fractional integrals and derivatives. Equations (3.56) and (3.57) express the suggested derivatives and integrals through the operators (3.50) and (3.51).

Example 1. Using equation 5.1 .7 of Pskhu, A. V. (2005), p. 136, we can define the Nakhushev fractional integral in the form

$I_{N}^{[\alpha, \beta]} X(t)=\frac{1}{\beta-\alpha} \cdot \int_{\alpha}^{\beta} I_{R L, a+}^{\xi} X(t) d \xi=\int_{0}^{t} W(\alpha, \beta, t-\tau) \cdot X(\tau) d \tau$,

that is $I_{N}^{[\alpha, \beta]} X(t)=W(\alpha, \beta, t-\tau) * X(t)$, where we use the function

$W(\alpha, \beta, t-\tau):=\frac{1}{(\beta-\alpha) \cdot t} \cdot V i(\alpha, \beta, t)=\frac{1}{(\beta-\alpha) \cdot t} \cdot \int_{\alpha}^{\beta} \frac{t^{\xi} d \xi}{\Gamma(\xi)}$.

This operator allows us to define the multiplier with uniform distributed memory (the UDM multiplier) by the equation

$Y(t)=m([\alpha, \beta]) \cdot I_{N}^{[\alpha, \beta]} X(t)$,

where $I_{N}^{[\alpha, \beta]}$ is the Nakhushev fractional integral (3.58).

Example 2. Using equation 5.1.26 of Pskhu, A. V. (2005), p. 143, the Nakhushev fractional derivative can be written in the form

$D_{N}^{[\alpha, \beta]} X(t):=D^{n}(W(n-\alpha, n-\beta, t) * X(t))$,

where $\beta>\alpha>0$. Expression (3.61) can be rewritten as

$D_{N}^{[\alpha, \beta]} X(t)=\left(\frac{d}{d x}\right)^{n} \int_{0}^{t} W(n-\alpha, n-\beta, t-\tau) \cdot X(\tau) d \tau$,

where $\beta>\alpha>0$. Using the Nakhushev fractional derivative (3.62), the accelerator with uniform distributed memory (the UDM accelerator) can be defined by the equation

$Y(t)=a([\alpha, \beta]) \cdot D_{N}^{[\alpha, \beta]} X(t)$,

where $a([\alpha, \beta])$ is the accelerator coefficient, which depends on the interval $[\alpha, \beta]$.

Example 3. Using equation 5.1.7 of Pskhu, A. V. (2005), p. 136, the Pskhu fractional integral can be defined by the expression

$I_{P}^{[\alpha, \beta]} X(t)=(\beta-\alpha) \cdot\left(t^{\beta-1} \cdot E_{\beta-\alpha}\left[t^{\beta-\alpha} ; \beta\right]\right) * X(t)$,

where $\beta>\alpha>0$, which can be written in the form

$I_{P}^{[\alpha, \beta]} X(t)=(\alpha-\beta) \cdot \int_{0}^{t} \cdot E_{\beta-\alpha}\left[(t-\tau)^{\beta-\alpha} ; \beta\right] \cdot(t-\tau)^{\beta-1} \cdot X(\tau) d \tau$,

where $\beta>\alpha>0$. This integral operator allows us to define the multiplier with non-singular distributed memory (the NSDM multiplier) by the equation

$Y(t)=m([\alpha, \beta]) \cdot I_{P}^{[\alpha, \beta]} X(t)$,

where $I_{P}^{[\alpha, \beta]}$ is the Pskhu fractional integral (3.65).

Example 4. Using equation 5.1.7 of Pskhu, A. V. (2005), p. 136, we can define the Pskhu fractional derivative as

$D_{P}^{[\alpha, \beta]} X(t):=-(\beta-\alpha) \cdot D^{n}\left(\left(t^{-\alpha} \cdot E_{\beta-\alpha}^{n-1}\left[t^{\beta-\alpha} ; 1-\alpha\right]\right) * X(t)\right)$,

where $\beta>\alpha>0$ and $E_{\alpha}^{\mu}[z ; \beta]$ is defined by the equation

$E_{\alpha}^{\mu}[z ; \beta]:=\frac{\partial}{\partial \mu}\left(z^{\mu} \cdot E_{\alpha}[z ; \beta+\mu]\right)$.

As a result, we have

$D_{P}^{[\alpha, \beta]} X(t)=(\alpha-\beta) \cdot\left(\frac{d}{d x}\right)^{n} \int_{0}^{t} E_{\beta-\alpha}^{n-1}\left[(t-\tau)^{\beta-\alpha} ; 1-\alpha\right] \cdot(t-\tau)^{-\alpha} \cdot X(\tau) d \tau$, 
where $\beta>\alpha>0$. Using this fractional derivative, the accelerator with non-singular distributed memory (the NSDM accelerator) can be defined by the equation

$Y(t)=a([\alpha, \beta]) \cdot D_{P}^{[\alpha, \beta]} X(t)$,

where $D_{P}^{[\alpha, \beta]}$ is the Pskhu fractional derivative (3.68).

Note that the Nakhushev fractional derivatives cannot be considered as inverse operators for the Nakhushev fractional integration. The Pskhu fractional derivatives are inverse to the Nakhushev fractional integration and the Pskhu fractional integrals are inverse to the Nakhushev fractional derivatives.

\subsection{Principle of Violation of Superposition for Accelerators with Memory}

Let us consider how the sequential action of two accelerators with power memory, whose fading parameters are equal to $\alpha>0$ and $\beta>0$, and the effect of the accelerator, whose fading parameter is equal to the sum of these parameters, $\alpha+\beta$.

Principle of violation of superposition for accelerators with memory: In the general case, the action of the accelerators with power-law memory cannot be equivalent to the action of an accelerator with power-law memory. Symbolically, this principle can be written in the form of the inequality

$E_{0}^{t ; \alpha}\left(E_{0}^{t ; \beta}(X(\tau))\right) \neq E_{0}^{t ; \alpha+\beta}(X(\tau))$

for $\alpha>0$ and $\beta>0$.

The principle of violation of superposition of accelerators with memory means that in general we have the inequality (3.70). As a result, the action of two accelerators with power-law memory

$Y(t)=E_{0}^{t ; \alpha}(X(\tau))$
$Z(t)=E_{0}^{t ; \beta}(Y(\tau))$

is not equivalent to the action of the accelerator with memory

$Z(t)=E_{0}^{t ; \alpha+\beta}(X(\tau))$

where the fading parameters $\alpha$ and $\beta$ satisfy the condition $\alpha>\beta>0$.

Example 1. The action of the multiplier with simplest power-law memory

$Y(t)=a(\alpha) \cdot D_{R L, 0+}^{\alpha}(X(\tau))$

and the accelerator with simplest power-law memory

$Z(t)=a(\beta) \cdot D_{R L, 0+}^{\beta}(Y(\tau))$

is equivalent to the action of the multiplier with memory

$Z(t)=a(\alpha, \beta) \cdot D_{R L, a+}^{\alpha+\beta}(X(\tau))$

where $a(\alpha, \beta)=a(\alpha) \cdot a(\beta)$ if fading parameters $\alpha$ and $\beta$ satisfy the condition where $\alpha>\beta>0$.

In the case of the accelerators with memory, which is described by the Riemann-Liouville fractional derivatives, the violation of superposition is based on the following property of the Riemann-Liouville fractional derivatives (see Property 2.4 of Kilbas, A. A., et. al. (2006), p. 75). Let $\alpha>0$ and $\beta>0$ be such that $n-1<\alpha \leq n$, $m-1<\beta \leq m$ and $\alpha+\beta<n$ and let $X(t) \in L_{1}(a, b)$ with $p \geq 1$, and $I_{R L, a+}^{m-\alpha} X(t) \in A C^{m}[a, b]$. Then we have (see Property 2.4 of Kilbas, A. A., et. al. (2006), p. 75) the equality

$D_{R L, a^{+}}^{\alpha} D_{R L, a^{+}}^{\beta} X(t)=D_{R L, a+}^{\alpha+\beta} X(t)-\sum_{k=1}^{m} \frac{(t-a)^{-\alpha-k}}{\Gamma(1-k-\alpha)} \cdot\left(D_{R L, a+}^{\beta-k} X\right)(a+)$.

Equation (3.77) means that, in general, we have the inequality

$D_{R L, a^{+}}^{\alpha} D_{R L, a^{+}}^{\beta} X(t) \neq D_{R L, a^{+}}^{\alpha+\beta} D_{R L, a^{+}}^{\alpha} X(t)$,

For the Caputo fractional derivative, we have the inequality

$D_{C, a^{+}}^{\alpha} D_{C, a+}^{\beta} X(t) \neq D_{C, a^{+}}^{\beta} D_{C, a^{+}}^{\alpha} X(t)$.

The same properties we have for the other type of fractional derivatives of non-integer orders.

This principle of violation of superposition leads to the following consequence that state the non-permutation (the noncommutativity) of actions of accelerators with memory. 
Principle of non-permutation of accelerator actions: In the general case, the accelerator action depends on their action sequences. Symbolically, this principle can be written in the form of the inequality

$E_{0}^{t ; \alpha}\left(E_{0}^{t ; \beta}(X(\tau))\right) \neq E_{0}^{t ; \beta}\left(E_{0}^{t ; \alpha}(X(\tau))\right)$

for $\alpha>0$ and $\beta>0$.

For the accelerators (3.74) and (3.75), this principle, which describes a dependence of the accelerator effect on sequence in the case of power-law memory, is based on the inequality that describes non-commutativity of fractional derivatives. For Riemann-Liouville derivatives, we have

$D_{R L, a^{+}}^{\alpha} D_{R L, a+}^{\beta} X(t) \neq D_{R L, a^{+}}^{\beta} D_{R L, a+}^{\alpha} X(t)$.

For the Caputo fractional derivative, we have the inequality

$D_{C, a^{+}}^{\alpha} D_{C, a^{+}}^{\beta} X(t) \neq D_{C, a^{+}}^{\beta} D_{C, a+}^{\alpha} X(t)$.

The same properties are satisfied for other type of fractional derivatives of non-integer orders.

\subsection{Superposition of Accelerators with Memory and without Memory}

This principle describes superposition for memory fading of the accelerator with memory and the standard accelerator without memory.

Principle of superposition for accelerators with memory and without memory: In the general case, the action of the accelerators with power-law memory and the accelerator without memory can be equivalent to the action of an accelerator with power-law memory.

This superposition principle for accelerator means that we have the equality

$E_{0}^{t ; \alpha}\left(E_{0}^{t ; \beta}(X(\tau))\right)=E_{0}^{t ; \alpha+\beta}(X(\tau))$

if at least one of the parameters $\alpha>0$ or $\beta>0$ takes the positive integer value. Implementation of the superposition for the left-sided or right-sided actions of the standard accelerators depends on the type of dynamic memory. This principle is based on the corresponding properties of fractional derivatives.

If the fractional derivatives $\left(D_{R L, a+}^{\alpha} X\right)(t)$ and $\left(D_{R L, a+}^{\alpha+k} X\right)(t)$ exist, where $\alpha \geq 0$ and $k$ is positive integer, then the following equality (see Property 2.3 of Kilbas, A. A., et. al. (2006), p. 74) is satisfied

$D^{k} D_{R L, a+}^{\alpha} X(t)=D_{R L, a}^{\alpha+k} X(t)$.

For details see Equation 2.144 of Podlubny, I. (1998), p. 81. This property leads to the superposition for the left-sided action of the standard accelerator.

If the fractional derivatives $\left(D_{C, a^{+}}^{\alpha} X\right)(t)$ and $\left(D_{C, a^{+}}^{\alpha+k} X\right)(t)$ exist, where $\alpha>0$ and $k$ is positive integer number, then we have the equality

$D_{C, a^{+}}^{\alpha} D^{k} X(t)=D_{C, a^{+}}^{\alpha+k} X(t)$.

In particular, if $k=1$, then we have $D_{C, a^{+}}^{\alpha} D^{1} X(t)=D_{C, a^{+}}^{\alpha+1} X(t)$. For details see Equation 2.143 of Podlubny, I. (1998), p. 81. This property leads to the superposition for the right-sided action of the standard accelerator.

If the Liouville fractional derivatives $\left(D_{L,+}^{\alpha} X\right)(t)$ and $\left(D_{L,+}^{\alpha+k} X\right)(t)$ exist, where $\alpha>0$ and $k$ is positive integer number, then we have the equality in the form

$D^{k} D_{L,+}^{\alpha} X(t)=D_{L,+}^{\alpha+k} X(t)$.

For details see Property 2.13 of Kilbas, A. A., et. al. (2006), p. 89.

For equalities (3.84), (3.85) and (3.86) the left and right positions of the accelerator with memory are important.

\subsection{Chain Rule and Product Rule for Accelerator with Memory}

It is known that the standard chain rule (the rule of differentiation of a composite function) can be used for the standard linear accelerators without memory. Let us consider the standard accelerator equations 


$$
Y(t)=a \cdot \frac{d X(t)}{d t} .
$$

If we consider the exogenous variable as the composition of two functions $X(t)=X_{1}\left(X_{2}(t)\right)$, then we have the equality

$$
Y(t)=a \cdot \frac{d X_{1}\left(X_{2}(t)\right)}{d t}=a \cdot \frac{\partial X_{1}}{\partial X_{2}} \cdot \frac{d X_{2}(t)}{d t}=\frac{\partial X_{1}}{\partial X_{2}} \cdot Y_{2}(t)
$$

for wide class of functions $X_{1}(t)$ and $X_{2}(t)$, where we use $Y_{2}(t)=a \cdot \frac{d X_{2}(t)}{d t}$.

It should be noted that the standard rule for differentiating a composite function does not hold for derivatives of non-integer order. As a result, analytical analysis of the non-linear relationships between $\mathrm{X}(\mathrm{t})$ and $\mathrm{Y}(\mathrm{t})$, as well as the composition of functions is significantly more complicated (see Podlubny, I. (1998), p. 97-98, and Tarasov, V. E. (2016b)). For example, the composition of functions is considered for the function $C=C(Y(t))$, which describes the dependence of consumption on income, Allen, R. G. D. (1959).

It is known that the standard product rule (the rule of differentiation of the product of functions) can be used for standard linear accelerator without memory. Let us consider the standard accelerator equations. If we consider the exogenous variable $X(t)=X_{1}(t)$ and $X(t)=X_{2}(t)$, then we have

$Y_{1}(t)=a \cdot \frac{d X_{1}(t)}{d t}, Y_{2}(t)=a \cdot \frac{d X_{2}(t)}{d t}$.

If we consider the exogenous variable $X(t)=X_{1}(t) \cdot X_{2}(t)$, then the action of the standard accelerator satisfies the product rule

$Y_{12}=a \cdot \frac{d}{d t}\left(X_{1}(t) \cdot X_{2}(t)\right)=a \cdot \frac{d X_{1}(t)}{d t} \cdot X_{2}(t)+a \cdot X_{1}(t) \cdot \frac{d X_{2}(t)}{d t}=$

$Y_{1}(t) \cdot X_{2}(t)+X_{1}(t) \cdot Y_{2}(t)$

for wide class of functions $X_{1}(t)$ and $X_{2}(t)$.

For the accelerators with power-law memory this standard product rule (3.81) is violated (see Tarasov, V. E. $(2013,2016 \mathrm{a}))$ since the standard product rule is violated for the fractional derivatives of non-integer orders $D_{C, a^{+}}^{\alpha}\left(X_{1}(t) \cdot X_{2}(t)\right) \neq D_{C, a^{+}}^{\alpha}\left(X_{1}(t)\right) \cdot X_{2}(t)+X_{1}(t) \cdot D_{C, a^{+}}^{\alpha}\left(X_{1}(t) \cdot X_{2}(t)\right)$.

This property should be taken into account in applications of the concepts of accelerator with power-law memory.

\section{Duality of Multiplier with Memory and Accelerator with Memory}

\subsection{Duality Principle}

Let us consider two variables $\mathrm{X}(\mathrm{t})$ and $\mathrm{Y}(\mathrm{t})$, that are functions of time. The function $\mathrm{X}(\mathrm{t})$, which describes an exogenous variable, is considered as the impact (or perturbation). The function $\mathrm{Y}(\mathrm{t})$, which describes an endogenous variable, is considered as a response (reaction) to the impact. In the general case, the equation of the generalized multiplier with memory

$Y(t)=F_{0}^{t}(X(\tau))$

is irreversible. This irreversibility means that there is no inverse operator $G_{0}^{t}$, such that

$X(t)=G_{0}^{t}(Y(\tau))$

for all $t>0$. In other words, knowing the function $\mathrm{Y}(\mathrm{t})$ on the interval $(0, t)$, it is not always possible to determine uniquely the function $\mathrm{X}(\mathrm{t})$ from equation (4.1). In some cases, the inverse operator $G_{0}^{t}$ exists, which is inversed from the left to multiplier equation (4.1), and equation (4.2) can be considered as an equation of accelerator with memory. Symbolically, this can be written in the form of the equality

$G_{0}^{t}\left(F_{0}^{t}(X(\tau))\right)=X(t)$

that holds for a wide class of functions $X(t)$. If condition (4.3) is realized then equation (4.2) can be interpreted as an equation of accelerator with memory. In this case, the accelerator with memory (4.2.) will be called dual to the multiplier with memory (4.1.).

Equation (4.3) means that the operator $G_{0}^{t}$ is the inverse of the left for the operator $F_{0}^{t}$. In the general case, the operator $G_{0}^{t}$ cannot be considered as a right inverse to the operator $F_{0}^{t}$, i.e., we have the inequality $F_{0}^{t}\left(G_{0}^{t}(Y(\tau))\right) \neq Y(t)$ in general. This inequality means that substitution of (4.2) into equation (4.1) does not 
result in the identity. This is due to the fact that equations (4.1) and (4.2) are satisfied for different function spaces.

Example: Let us consider the linear multiplier with simplest power-law memory. In this case, the equation of multiplier with memory (4.1), which has the form $Y(t)=m(\alpha) \cdot\left(I_{R L ; 0+}^{\alpha} X\right)(t)$, is reversible such that the dual (inverse) equation has the form $X(t)=a(\alpha) \cdot D_{R L ; 0+}^{\alpha} Y(t)$, where $a(\alpha)=1 / m(\alpha)$, which can be considered as an equation of accelerator with memory. In general, we have the inequality $I_{R L ; 0+}^{\alpha} D_{R L ; 0+}^{\alpha} Y(t) \neq Y(t)$.

In the general case, the equation of the generalized accelerator with memory

$Y(t)=E_{0}^{t}(X(\tau))=F_{0}^{t}\left(X^{(n)}(\tau)\right)$

is not reversible and there is no inverse operator $H_{n ; 0}^{t}$ such that

$X(t)=H_{n ; 0}^{t}(Y(\tau))+b_{X ; 0+}(t)$,

where $b_{X ; 0+}(t)$ is a function of time $\mathrm{t}$, which is defined by some properties of the function $\mathrm{X}(\mathrm{t})$ and the integer derivatives $X^{(k)}(\tau)$ of the orders $k=1,2, \ldots, n$ at zero $(t \rightarrow 0+)$. It should be noted that in some cases, the inverse operator $H_{n ; 0}^{t}$ exists and equation (4.5), which is inverse to accelerator equation (4.4), can be considered as an equation of multiplier with memory. Symbolically, this can be written in the form of the equality

$H_{n ; 0}^{t}\left(F_{0}^{t}\left(X^{(n)}(\tau)\right)\right)=X(t)-b_{X ; 0+}(t)$

that holds for a wide class of functions $X(t)$. Here $b_{X ; 0+}(t)$ means an additional term that depends on $\mathrm{X}(\mathrm{t})$ and their derivatives at $t \rightarrow 0+$. In many cases, we also have the equality

$E_{0}^{t}\left(H_{n ; 0}^{t}(Y(\tau))+b_{X ; 0+}(t)\right)=Y(t)$.

This equation means that substitution of (4.5) into equation (4.4) gives the identity.

Example: Let us consider the linear accelerator with simplest power-law memory. In this case, the equation of multiplier with memory (4.1), which has the form $Y(t)=a(\alpha) \cdot\left(D_{C ; 0+}^{\alpha} X\right)(t)$, is reversible such that the dual (inverse) equation has the form $X(t)=m(\alpha) \cdot I_{R L ; 0+}^{\alpha} Y(t)+b_{X}(t)$, where $m(\alpha)=1 / a(\alpha), n-1<\alpha<n$, and $b_{X}(t)=\sum_{k=0}^{n-1} \frac{X^{(k)}(0) \cdot t^{k}}{\Gamma(k+1)}$, which can be interpreted as a linear multiplier simplest power-law memory. In this case, we have the equality

$a(\alpha) \cdot\left(D_{C ; 0+}^{\alpha}\left(m(\alpha) \cdot\left(I_{R L ; 0+}^{\alpha} Y\right)(\tau)+b(\tau)\right)\right)(t)=Y(t)$.

Let us formulate a general duality principle, which states a relationship between the concepts of the accelerator with memory and the multiplier with memory.

General duality principle. The principle of duality of the linear accelerator with memory and the linear multiplier with memory states a possibility to represent equation of the multiplier by equations of the accelerator together with a possibility to represent this accelerator equation by equation of a multiplier with memory. In other words, the principle of duality of the accelerator with memory and the multiplier with memory gives the following relationship of these concepts.

$[M \rightarrow A]$ The equation of multiplier with memory is a reversible such that the dual (inverse) equation is an equation of accelerator with memory;

$[A \rightarrow M]$ The equation of accelerator with memory is reversible such that the dual (inverse) equation can be interpreted as the equations of the multiplier with memory.

In the transformations between the accelerator and multiplier with memory, the exogenous and endogenous variables, which are described by the functions with continuous time, actually change the roles. In other words, when describing memory effects, the transition from multiplier with memory to accelerator with memory is accompanied by the fact that endogenous variables should be considered as exogenous and conversely.

A detailed description of different examples and it proofs will be given in the next sections.

Remark 1. Let us consider the linear generalized multiplier with memory of the special kind that is described by the equation

$Y(t)=\int_{0}^{t} M(t, \tau) \cdot X(\tau) d \tau$ 
where $M(t, \tau)$ is the memory function. In equation (4.10) we can consider $\mathrm{Y}(\mathrm{t})$ as a given function, and the variable $\mathrm{X}(\mathrm{t})$ can be considered as the unknown function. In this case, equation (4.9) is called the linear Volterra equations of the first kind. Existence of solutions of equation (4.9), i.e. the reversibility of equation (4.9), has been studied in the theory of integral equations.

Remark 2. Let us consider the linear generalized accelerator with memory that is described by the equation

$$
Y(t)=\int_{0}^{t} M(t, \tau) \cdot X^{(n)}(\tau) d \tau
$$

where $M(t, \tau)$ is the memory function. In equation (4.10) we can consider $\mathrm{Y}(\mathrm{t})$ as a given function, and the variable $\mathrm{X}(\mathrm{t})$ can be considered as the unknown function. In this case equation (4.10) can be associated with the following inverse problems of dynamics (for example, see Galiullin, A. S. (1984), Sverdlove R. (1981), Gladwell, G. M. L., Morassi, A. (2011)), which is the economic dynamics in our case. For example, if we know the memory function $M(t, \tau)$ of the economic process and endogenous variable $\mathrm{Y}(\mathrm{t})$, then we can put the problem of finding (the restoration) of the impact function (the exogenous variables $\mathrm{X}(\mathrm{t})$ ) for this process. This type of problems includes finding solutions of the equations of macroeconomic growth models with memory.

In the next section, we consider the duality principle for accelerator and multiplier with simplest power memory with details. Then the duality principle is demonstrated for different type of dynamic memory.

\subsection{Duality for Accelerator and Multiplier with Simplest Power-Law Memory}

\subsubsection{From Multiplier with Memory to Accelerator with Memory}

Let us consider now the multiplier with simplest power-law memory, which is described by the equation $Y(t)=m(\alpha) \cdot\left(I_{R L ; 0+}^{\alpha} X\right)(t)$, where $\alpha$ is an arbitrary positive number that characterized the memory fading. We can use the property, which states that the Caputo derivative is the inverse operation to the Riemann-Liouville integral. This property leads to the important statement about the relationship between the concepts of the multiplier and the accelerator with memory.

Principle of duality of multiplier and accelerator with power-law memory. Part $1[M \rightarrow A]:$ Let $X(\tau)$ be the function of the factor, which is continuous on the finite interval $[0, t]$, i.e., $X(\tau) \in C[0, t]\left(\right.$ or $\left.X(\tau) \in L_{\infty}(0, t)\right)$. Then the indicator $Y(t)$, which is described by the equation of linear multiplier with memory $Y(t)=m(\alpha) \cdot\left(I_{R L ; 0+}^{\alpha} X\right)(t)$,

where $m(\alpha)$ is the multiplier coefficient and $\alpha>0$, satisfies the equation of accelerator with memory

$X(t)=a(\alpha) \cdot\left(D_{C ; 0+}^{\alpha} Y\right)(t)$,

where $a(\alpha)=1 / m(\alpha)$ is the accelerator coefficient. In the duality concepts exogenous and endogenous variables actually change the roles.

Proof. To prove the statement of this principle, we apply the Caputo derivative of order $\alpha>0$ to equation (4.11). As a result, we obtain the expression

$\left(D_{C ; 0+}^{\alpha} Y\right)(t)=m(\alpha) \cdot\left(D_{C ; 0+}^{\alpha} I_{R L ; 0+}^{\alpha} X\right)(t)$.

Let us use Lemma 2.21 of Kilbas, A .A., et. al. (2006), p. 95, which states that the Caputo fractional derivative is the inverse operation to the Riemann-Liouville fractional integral. According to this Lemma, if $X(t) \in C[a, b]$ or $X(t) \in L_{\infty}(a, b)$, then the identity

$\left(D_{C ; 0+}^{\alpha} I_{R L ; 0+}^{\alpha} X\right)(t)=X(t)$

is satisfied for all $\alpha>0$, where $D_{C ; 0+}^{\alpha}$ is the left-sided Caputo fractional derivative (3.25) of the order $\alpha>0$, and $I_{R L ; 0+}^{\alpha}$ is the left-sided Riemann-Liouville fractional integral (2.25) of the order $\alpha>0$.

Using equation (4.14) for the right-hand side of equation (4.13), we obtain

$\left(D_{C ; 0+}^{\alpha} Y\right)(t)=m(\alpha) \cdot X(t)$.

Using the coefficient $a(\alpha)=1 / m(\alpha)$, equation (4.15) is written in the form (4.12), which is the accelerator equation of the same order $\alpha>0$. This is the end of the proof. 
As a result, we proved that the equations of accelerator with memory of an arbitrary non-negative order $\alpha$ can be derived from the multiplier equation with memory of same order $\alpha$. In equation (4.11) the variable $\mathrm{X}(\mathrm{t})$ is exogenous and $\mathrm{Y}(\mathrm{t})$ is endogenous variable. In equation (4.12) the variable $\mathrm{Y}(\mathrm{t})$ is exogenous and $\mathrm{X}(\mathrm{t})$ is endogenous variable.

\subsubsection{From Accelerator with Memory to Multiplier with Memory}

Let us consider the accelerator equation with simplest power-law memory, for which the parameter $\alpha>0$ characterizes the memory fading.

Principle of duality of multiplier and accelerator with power-law memory. Part $2[A \rightarrow M]:$ Let $X(\tau)$ be the function of the factor, which has derivatives up to (n-1)th order that are continuous functions on the interval $[0, t]$, i.e., $X(\tau) \in C^{n}[0, t]$. Then the indicator $Y(t)$, which is described by the equation of linear accelerator with memory

$Y(t)=a(\alpha) \cdot\left(D_{C ; 0+}^{\alpha} X\right)(t)$,

where $a(\alpha)$ is the accelerator coefficient and $0 \leq n-1<\alpha<n$, satisfies the equation of the multiplier with memory

$X(t)=m(\alpha) \cdot I_{0+}^{\alpha} Y(t)+b(t)$

where $m(\alpha)=1 / a(\alpha)$ is the multiplier coefficient, and

$b(t)=\sum_{k=0}^{n-1} X^{(k)}(0) \cdot \frac{t^{k}}{\Gamma(k+1)}$.

It should be noted that the substitution of (4.17) and (4.18) into equation (4.16) gives the identity. In the duality concepts exogenous and endogenous variables actually change the roles.

Proof. Using the Riemann-Liouville integral, the integration of equation (4.17) gives

$I_{R L ; 0+}^{\alpha} Y(t)=a(\alpha) \cdot\left(I_{R L ; 0+}^{\alpha} D_{C ; 0+}^{\alpha} X\right)(t)$.

If the function $X(t)$ is continuously differentiable $n$ times, then using Lemma 2.2 of Kilbas, A .A., et. al. (2006), p. 95, we have the equality

$\left(I_{R L ; 0+}^{\alpha} D_{C ; 0+}^{\alpha} X\right)(t)=X(t)-\sum_{k=0}^{n-1} X^{(k)}(0) \cdot \frac{t^{k}}{\Gamma(k+1)}$.

where $n-1<\alpha<n$. Substituting equation (4.20) into equation (4.19), we obtain

$I_{R L ; 0+}^{\alpha} Y(t)=a(\alpha) \cdot X(t)-a(\alpha) \cdot \sum_{k=0}^{n-1} X^{(k)}(0) \cdot \frac{t^{k}}{\Gamma(k+1)}$.

Using $m(\alpha)=1 / a(\alpha)$, equation (4.21) gives (4.17). This is the end of the proof.

Equation (4.15) describes a linear multiplier with memory. As a result, the linear accelerator equation with memory is equivalent to the linear equation of the multiplier with memory, in which the indicator $\mathrm{Y}(\mathrm{t})$ and factor $\mathrm{X}(\mathrm{t})$ are exchanged places and $m(\alpha)=1 / a(\alpha)$.

For $0<\alpha<1$, the free term $b(t)=X(0)$. As a result, if $X(0)=0$ and $0<\alpha<1$, then we get the equation of the multiplier with memory in the form $X(t)=m(\alpha) \cdot I_{R L ; 0+}^{\alpha} Y(t)$. The same equation can be derived if $X^{(k)}(0)=0$ for all $k=0,1, \ldots n-1$, where $0 \leq n-1<\alpha<n$.

In equation (4.17) the variable $\mathrm{X}(\mathrm{t})$ is exogenous and $\mathrm{Y}(\mathrm{t})$ is endogenous variable. In equation (4.18) the variable $\mathrm{Y}(\mathrm{t})$ is exogenous and $\mathrm{X}(\mathrm{t})$ is endogenous variable. In the transformations between these notions, the exogenous and endogenous variables actually change the roles. In other words, when describing memory effects, the transition from multiplier with memory to accelerator with memory is accompanied by the fact that endogenous variables should be considered as exogenous and, conversely. Accelerator coefficients with memory are defined as the inverse value of multiplier coefficients with memory. If the coefficient of the multiplier with memory is greater than one, then the coefficient of the dual accelerator will be less than one.

We consider the duality of the accelerator with memory and the multiplier with memory only for one-parametric memory fading. 
As a result, the described relationship between equations (4.13) and (4.14), and also between equations (4.17) and (4.18), actually means a duality of the concepts of accelerator and multiplier with memory.

\subsubsection{Duality Principle for Accelerator and Multiplier with Simplest Power-Law Memory}

Part $1[M \rightarrow A]$ and Part $2[A \rightarrow M]$ of the principle of duality of multiplier and accelerator can be combined into one principle of duality. This principle describes the mutual duality of the concepts of accelerator and multiplier with simplest power-law memory.

Let us consider the accelerator with power-law memory $Y(t)=a(\alpha) \cdot\left(D_{C ; 0+}^{\alpha} X\right)(t)$, which is described by the Caputo fractional derivative, and the multiplier with memory $Y(t)=m(\alpha) \cdot\left(I_{R L ; 0+}^{\alpha} X\right)(t)$ that is described by the Riemann-Liouville integral. The concepts of the multiplier and accelerator with simplest power-law memory are dual to each other. Let us formulate the principle of duality for these concepts.

First Principle of duality of multiplier and accelerator with simplest power-law memory:

$[M \rightarrow A]$ The multiplier with power-law memory, which is described by the equation

$Y(t)=m(\alpha) \cdot I_{R L, 0+}^{\alpha} X(t)$,

can be described as the accelerator with memory

$X(t)=a(\alpha) \cdot D_{C, 0+}^{\alpha} Y(t)$

where $a(\alpha)=\frac{1}{m(\alpha)}$ is the coefficient of the dual accelerator, and the exogenous and endogenous variables actually change the roles.

$[A \rightarrow M]$ The accelerator with power-law memory, which is described by the equation

$Y(t)=a(\alpha) \cdot D_{C, 0+}^{\alpha} X(t)$,

can be described as the accelerator with memory

$X(t)=m(\alpha) \cdot I_{R L, 0+}^{\alpha} Y(t)+b_{X}(t)$.

where $m(\alpha)=\frac{1}{a(\alpha)}$ is the coefficient of the dual multiplier,

$b_{X}(t)=\sum_{k=0}^{n-1} p_{k}^{C}(0) \cdot t^{k}$

with $p_{k}^{C}(0)=\frac{X^{(k)}(0)}{k !}$. In the duality concepts exogenous and endogenous variables actually change the roles.

The proof of the duality of the accelerator and multiplier with power-law memory is based on the Lemma 2.21 and Lemma 2.22 of Kilbas, A. A., et. al. (2006), p. 95-96.

Lemma 2.21 states the following. If $\alpha>0$, then (see Lemma 2.21 of Kilbas, A. A., et. al. (2006), p. 95) the equality

$D_{C, a+}^{\alpha} I_{R L, a+}^{\alpha} X(t)=X(t)$

is valid for any function $X(t) \in L_{\infty}(a, b)$ or $X(t) \in C[a, b]$. Using (4.28), the action of the Caputo fractional derivative on equation (4.23) gives equation (4.24).

Lemma 2.21 states the following. Let $\alpha>0, n=[\alpha]+1$ for non-integer $\alpha$ and $n=\alpha$ for integer $\alpha$. If $X(t) \in A C^{n}[a, b]$ or $X(t) \in C^{n}[a, b]$, then (see Lemma 2.22 of Kilbas, A. A., et. al. (2006), p. 96) we have the equality

$I_{R L, a+}^{\alpha} D_{C, a+}^{\alpha} X(t)=X(t)-\sum_{k=0}^{n-1} p_{k}^{C}(a) \cdot(t-a)^{k}$,

where

$p_{k}^{C}(a)=\frac{X^{(k)}(a)}{k !}$.

In particular, for $0<\alpha<1(n=1)$ the equation

$I_{R L, a+}^{\alpha} D_{C, a+}^{\alpha} X(t)=X(t)-X(a)$

is valid if $X(t) \in A C[a, b]$ or $X(t) \in C[a, b]$. Using (4.28), the action of the Riemann-Liouville fractional integration on equation (4.24) gives equation (4.25). 


\subsection{New Examples of Duality for Accelerator and Multiplier with Memory}

\subsubsection{Second duality for Accelerator and Multiplier with Simplest Power-Law Memory}

In the previous section, we consider accelerator that is described by the Caputo fractional derivatives. Let us consider the accelerator with power-law memory $Y(t)=a(\alpha) \cdot\left(D_{R L ; 0+}^{\alpha} X\right)(t)$, which is described by the Riemann-Liouville fractional derivatives instead of the Caputo fractional derivatives. The multiplier with memory will be described by the equation $Y(t)=m(\alpha) \cdot\left(I_{R L ; 0+}^{\alpha} X\right)(t)$, with the Riemann-Liouville integrals. The multiplier and accelerator with simplest power-law memory are dual concepts. Let us formulate the principle of duality for these concepts.

Second Principle of duality of multiplier and accelerator with power-law memory:

$[M \rightarrow A]$ The multiplier with power-law memory, which is described by the equation

$Y(t)=m(\alpha) \cdot I_{R L, 0+}^{\alpha} X(t)$,

can be described as the accelerator with memory

$X(t)=a(\alpha) \cdot D_{R L, 0+}^{\alpha} Y(t)$

where $a(\alpha)=\frac{1}{m(\alpha)}$ is the coefficient of the dual accelerator and the exogenous and endogenous variables actually change the roles.

$[A \rightarrow M]$ The accelerator with power-law memory, which is described by the equation

$Y(t)=a(\alpha) \cdot D_{R L, 0+}^{\alpha} X(t)$,

can be described as the accelerator with memory

$X(t)=m(\alpha) \cdot I_{R L, 0+}^{\alpha} Y(t)+b_{X}(t)$

where $m(\alpha)=\frac{1}{a(\alpha)}$ is the coefficient of the dual multiplier,

$b_{X}(t)=\sum_{k=0}^{n-1} p_{k}^{R L}(0) \cdot t^{\alpha-k-1}$

and

$p_{k}^{R L}(0)=\frac{\left(D^{n-k-1} I_{R L, a+}^{n-\alpha} X\right)(0)}{\Gamma(\alpha-k)}$.

In the duality concepts exogenous and endogenous variables actually change the roles.

Proof. The proof of the duality of the accelerator and multiplier with power-law memory is based on Theorem 2.4. of Samko, S. G. et. al. (1993), p. 44-45, (and Lemma 2.4 of Kilbas, A. A., et. al. (2006), p. 74) and Theorem 2.4. of Samko, S. G. et. al. (1993), p. 44-45 (and Lemma 2.5 of Kilbas, A. A., et. al. (2006), p. 74-75). The Riemann-Liouville fractional derivative provides operation inverse to the Riemann-Liouville integration from the left.

Using Theorem 2.4. of Samko, S. G. et. al. (1993), p. 44-45, we have that the equality $D_{R L, a^{+}}^{\alpha} I_{R L, a+}^{\alpha} X(t)=X(t)$

is valid for any summable function $\left(X(t) \in L_{1}(a, b)\right)$, where $D_{R L, 0+}^{\alpha}$ is the left-sided Riemann-Liouville fractional integral of the order $\alpha>0$, and $I_{R L, 0+}^{\alpha}$ is the left-sided Riemann-Liouville fractional integral of the order $\alpha>0$ with respect to the variable t. If $X(t) \in L_{p}(a, b)$ with $p \geq 1$, then (Lemma 2.4 of Kilbas, A. A., et. al. (2006), p. 74) equality (4.37) holds almost everywhere on $[a, b]$. Using (4.37), the action of the Riemann-Liouville fractional derivative on equation (4.32) gives equation (4.33).

If the function $X(t) \in L_{1}(a, b)$ has a summable fractional derivative $D_{R L, a+}^{\alpha} X(t)$, i.e. $I_{R L, a+}^{n-\alpha} X(t) \in A C^{n}[a, b]$, then (see Theorem 2.4. of Samko, S. G. et. al. (1993), p. 44-45, and Lemma 2.5 of Kilbas, A. A., et. al. (2006), p. 74-75) we have the equality

$I_{R L, a^{+}}^{\alpha} D_{R L, a^{+}}^{\alpha} X(t)=X(t)-\sum_{k=0}^{n-1} p_{k}^{R L}(a) \cdot(t-a)^{\alpha-k-1}$,

where $n=[\alpha]+1$ and

$p_{k}^{R L}(a)=\frac{1}{\Gamma(\alpha-k)} \cdot\left(D^{n-k-1} I_{R L, a+}^{n-\alpha} X\right)(a)$.

In particular, we have

$I_{R L, a+}^{\alpha} D_{R L, a^{+}}^{\alpha} X(t)=X(t)-\frac{(t-a)^{\alpha-1}}{\Gamma(\alpha)} \cdot\left(I_{R L, a+}^{1-\alpha} X\right)(a)$ 
for $0<\alpha<1(n=1)$. Using (4.38), the action of the Riemann-Liouville fractional integration on equation (4.34) gives equation (4.35).

\subsubsection{Duality for Accelerator and Multiplier with Parametric Memory}

Parametric fractional Caputo derivative has been suggested by equation (23) of Definition 3 of the paper by Tarasova, V. V., Tarasov, V. E. (2016b), p. 224. The properties of this derivative have been described by Almeida, R. (2017), p. 467.

The duality of the accelerator and multiplier with parametric memory is based on Theorems 4 and 5 of Almeida, R. (2017), p. 466-467. Let us give the statements of these theorems.

If $\alpha>0$ then (Theorem 5 of Almeida, R. (2017), p. 467) the equality

$D_{C, a+}^{\alpha, x} I_{R L, a+}^{\alpha, x} X(t)=X(t)$

is valid for any function $X(t) \in C^{1}[a, b]$.

If $X(t) \in C^{n}[a, b]$, then (Theorem 4 of Almeida, R. (2017), p. 466) we have the equality

$I_{R L, a^{+}}^{\alpha, x} D_{C, a^{+}}^{\alpha, x} X(t)=X(t)-\sum_{k=0}^{n-1} \frac{X^{(k)}(a)}{k !} \cdot(x(t)-x(a))^{k}$,

where $\alpha>0, n=[\alpha]+1$ for non-integer $\alpha$ and $n=\alpha$ for integer $\alpha$.

\subsubsection{Duality for Accelerator and Multiplier with Generalized Power-Law Memory}

We can consider two cases where accelerator is described by the Erdelyi-Kober fractional derivatives of Caputo and Riemann-Liouville types.

Example 1. Let us consider the accelerator with generalized power-law memory (the GPLM accelerator), which is described by equation $Y(t)=a(\alpha) \cdot\left(D_{E K, a+;, \eta}^{\alpha} X\right)(t)$, with the Erdelyi-Kober fractional derivative of the Riemann-Liouville type, and the GPLM multiplier $Y(t)=m(\alpha) \cdot\left(I_{E K, a+; \sigma, \eta}^{\alpha} X\right)(t)$ that is described by the Erdelyi-Kober integral. The multiplier and accelerator with generalized power-law memory are dual concepts. The principle of duality for these concepts can be formulated in the following form.

First Principle of duality of the GPLM multiplier and accelerator:

$[M \rightarrow A]$ The GPLM multiplier, which is described by the equation

$Y(t)=m(\alpha) \cdot I_{E K, 0+; \sigma, \eta}^{\alpha} X(t)$,

can be described as the GPLM accelerator

$X(t)=a(\alpha) \cdot D_{E K, a+; \sigma, \eta}^{\alpha} Y(t)$,

where $a(\alpha)=\frac{1}{m(\alpha)}$ is the coefficient of the dual accelerator and the exogenous and endogenous variables actually change the roles.

$[A \rightarrow M]$ The GPLM accelerator, which is described by the equation

$Y(t)=a(\alpha) \cdot D_{E K, a+; \sigma, \eta}^{\alpha} X(t)$,

can be described as the GPLM multiplier

$X(t)=m(\alpha) \cdot I_{E K, 0+; \sigma, \eta}^{\alpha} Y(t)+b_{X}(t)$,

where $m(\alpha)=\frac{1}{a(\alpha)}$ is the coefficient of the dual multiplier,

$b_{X}(t)=\sum_{k=0}^{n-1} p_{k}^{E K}(0) \cdot t^{-\sigma(\eta+1+k)}$.

In the duality concepts exogenous and endogenous variables actually change the roles.

Proof. The proof of this principle of duality of the accelerator and multiplier with generalized power-law memory, which is described by the Erdelyi-Kober fractional integrals and derivatives, is based on Theorem 3.1. of Luchko, Yu., Trujillo, J. J. (2007), p. 255-258, and Equation 21 of Luchko, Yu., Trujillo, J. J. (2007), p. 254] (or Property 2.23 of Kilbas, A. A., et. al. (2006), p. 109). 
The Erdelyi-Kober fractional derivative provides operation inverse to the Erdelyi-Kober integration from the left. We can use Property 2.23 of Kilbas, A. A., et. al. (2006), p. 109 and Equation 21 of Luchko, Yu., Trujillo, J. J. (2007), p. 254], where $a=0$. If $\alpha>0$ and $0 \leq a<b$, then the equality

$\left(D_{E K, a+; \sigma, \eta}^{\alpha} I_{E K, a+; \sigma, \eta}^{\alpha} X\right)(t)=X(t)$

is valid for all $X(t) \in C_{\mu}$, where $\mu \geq-\sigma(\eta+1)$. Using (4.48), the action of the Erdelyi-Kober fractional derivative on equation (4.43) gives equation (4.44).

Let $n-1<\alpha \leq n, \mu \geq-\sigma(\eta+1)$ and $X(t) \in C_{\mu}^{n}$, where the space of functions $C_{\mu}^{n}$ consists of all functions $\mathrm{X}(\mathrm{t})$ with $t>0$, that can be represented in the form $X(t)=t^{p} \cdot f(t)$ with $p>\mu$ and $f(t) \in C^{n}([0, \infty))$. Then using Theorem 3.1. of Luchko, Yu., Trujillo, J. J. (2007), p. 255-258, the following relation between $t$ the Erdelyi-Kober fractional derivative and the Erdelyi-Kober fractional integral of the order $\alpha>0$ holds true

$\left(I_{E K, 0+; \sigma, \eta}^{\alpha} D_{E K, 0+; \sigma, \eta}^{\alpha} X\right)(t)=X(t)-\sum_{k=0}^{n-1} p_{k}^{E K}(0) \cdot t^{-\sigma(\eta+1+k)}$,

where $p_{k}^{E K}(0)$ are defined by the equation

$p_{k}^{E K}(0)=\frac{\Gamma(n-k)}{\Gamma(\alpha-k)} \lim _{t \rightarrow 0} t^{\sigma(\eta+1+k)} \prod_{j=k+1}^{n-1}\left(\frac{\tau}{\sigma} \frac{d}{d \tau}+\eta+j+1\right)\left(I_{E K, 0+; \sigma, \eta+\alpha}^{n-\alpha} X\right)(t)$.

The equations of Theorem 3.1 are similar to the corresponding equations for the Riemann-Liouville fractional integral and derivative

$\left(I_{R L, 0+}^{\alpha} D_{R L, 0+}^{\alpha} X\right)(t)=X(t)-\sum_{k=1}^{n} p_{k}^{R L}(0) \cdot t^{\alpha-k}$

where $p_{k}^{R L}(0)$ are defined by the equation

$p_{k}^{R L}(0)=\frac{t^{\alpha-k}}{\Gamma(\alpha-k+1)} \cdot \lim _{t \rightarrow 0} D_{R L, 0+}^{\alpha-k} X(t)$.

Using (4.49), the action of the Erdelyi-Kober fractional integration on equation (4.45) gives equation (4.46) that describes the linear multiplier with generalized power-law memory.

Example 2. Let us consider the accelerator with memory $Y(t)=a(\alpha) \cdot\left(D_{E K C, a+; \sigma, \eta}^{\alpha} X\right)(t)$, which is described by the Erdelyi-Kober fractional derivative of the Caputo type, and the multiplier with memory $Y(t)=m(\alpha) \cdot\left(I_{E K, a+; \sigma, \eta}^{\alpha} X\right)(t)$ that is described by the Erdelyi-Kober integral. These equations of multiplier and accelerator with generalized power-law memory describe dual concepts. The duality principle for these concepts can be formulated in the following form.

\section{Second Principle of duality of the GPLM multiplier and accelerator:}

$[M \rightarrow A]$ The GPLM multiplier with generalized power-law memory, which is described by the equation

$Y(t)=m(\alpha) \cdot I_{E K, 0+; \sigma, \eta}^{\alpha} X(t)$,

can be described as the accelerator with memory

$X(t)=a(\alpha) \cdot D_{E K, a+; \sigma, \eta}^{\alpha} Y(t)$,

where $a(\alpha)=\frac{1}{m(\alpha)}$ is the coefficient of the dual accelerator and the exogenous and endogenous variables actually change the roles.

$[A \rightarrow M]$ The accelerator with power-law memory, which is described by the equation

$Y(t)=a(\alpha) \cdot D_{E K C, a+; \sigma, \eta}^{\alpha} X(t)$,

can be described as the multiplier with memory

$X(t)=m(\alpha) \cdot I_{E K, 0+; \sigma, \eta}^{\alpha} Y(t)+b_{X}(t)$,

where $m(\alpha)=\frac{1}{a(\alpha)}$ is the coefficient of the dual multiplier,

$b_{X}(t)=\sum_{k=0}^{n-1} p_{k}^{E K C}(0) \cdot t^{-\sigma(\eta+1+k)}$.

In the duality concepts exogenous and endogenous variables actually change the roles.

Proof. For this case, when the accelerator with memory is described by the Caputo-type modification of the Erdelyi-Kober fractional derivative, the duality is based on Theorem 4.1. of Luchko, Yu., Trujillo, J. J. (2007), p. 260-261, and Theorem 4.3. of Luchko, Yu., Trujillo, J. J. (2007), p. 263-264. 
The Caputo-type modification of the Erdelyi-Kober fractional derivative is a left-inverse operator to the Erdelyi-Kober fractional integral. This property is valid for the functions from the functional space $C_{\mu}$, where $\mu \geq-\sigma(\eta+1)$. If $\alpha>0$ and $0 \leq a<b$, then using Theorem 4.3. of Luchko, Yu., Trujillo, J. J. (2007), p. 263-264, we have

$\left(D_{E K C, 0+; \sigma, \eta}^{\alpha} I_{E K, 0+; \sigma, \eta}^{\alpha} X\right)(t)=X(t)$

for all $X(t) \in C_{\mu}$, where $\mu \geq-\sigma(\eta+1)$. Using (4.58), the action of the Erdelyi-Kober fractional derivative on equation (4.53) gives equation (4.54).

Let $n-1<\alpha \leq n, \mu \geq-\sigma(\alpha+\eta+1)$ and $X(t) \in C_{\mu}^{n}$, where the space of functions $C_{\mu}^{n}$ consists of all functions $\mathrm{X}(\mathrm{t}), t>0$, that can be represented in the form $X(t)=t^{p} \cdot f(t)$ with $p>\mu$ and $f(t) \in C^{n}([0, \infty)$. Then using Theorem 4.1. of Luchko, Yu., Trujillo, J. J. (2007), p. 260-261, the following relation between the Caputo-type modification of the Erdelyi-Kober fractional derivative and the Erdelyi-Kober fractional integral of the order $\alpha>0$ holds true

$\left(I_{E K, 0+; \sigma, \eta}^{\alpha} D_{E K C, 0+; \sigma, \eta}^{\alpha} X\right)(t)=X(t)-\sum_{k=0}^{n-1} p_{k}^{E K C}(0) \cdot t^{-\sigma(\eta+1+k)}$,

where $p_{k}$ are defined by the equation

$p_{k}^{E K C}(0)=\lim _{t \rightarrow 0} t^{\sigma(\eta+1+k)} \prod_{j=k+1}^{n-1}\left(\frac{\tau}{\sigma} \frac{d}{d \tau}+\eta+j+1\right) X(t)$.

Using (4.59), the action of the Erdelyi-Kober fractional derivative on equation (4.55) gives equation (4.56).

Note that equations (4.58) and (4.59) are similar to the corresponding equations for the Riemann-Liouville fractional integral and the Caputo fractional derivative. We emphasize that the constants $p_{k}$ depend only on the ordinary derivatives of the function $\mathrm{X}(\mathrm{t})$ with some power weights and do not depend on the limit values of the fractional integrals at the point $t=0$.

\subsubsection{Duality of UDN Multiplier and NSDM Accelerator}

Let us consider the multiplier with uniform distributed memory (the UDM multiplier) that is described by the equation

$Y(t)=m([\alpha, \beta]) \cdot I_{N}^{[\alpha, \beta]} X(t)$,

where $I_{N}^{[\alpha, \beta]}$ is the Nakhushev fractional integral.

Principle of duality of UDN multiplier and NSDM accelerator:

$[M \rightarrow A]$ For the concept of multiplier (4.61), there is a dual concept of the accelerator with non-singular distributed memory, which is described by the equation

$X(t)=a([\alpha, \beta]) \cdot D_{P}^{[\alpha, \beta]} Y(t)$,

where $D_{P}^{[\alpha, \beta]}$ is the Pskhu fractional derivative of the non-singular distributed order.

$[A \rightarrow M]$ For the concept of the accelerator with non-singular distributed memory

$Y(t)=a([\alpha, \beta]) \cdot D_{P}^{[\alpha, \beta]} X(t)$,

there is a dual concept of the multiplier with uniform distributed memory that is described by the equation

$X(t)=m([\alpha, \beta]) \cdot I_{N}^{[\alpha, \beta]} Y(t)+b_{X}(t)$,

where

$b_{X}(t)=\sum_{k=1}^{n} W(\alpha-k, \beta-k, t) \cdot\left(D_{P}^{[\alpha-k, \beta-k]} X\right)(0+)$.

In the dual concepts the exogenous and endogenous variables change the roles.

Proof. The proof of this duality is based on Theorem 1 of Pskhu, A. V. (2004) or Theorem 5.1.1 of Pskhu, A. V. (2005), p. 137-140, which can be written by the following statement. Let $\beta>\alpha>0$ and $n-1 \leq \alpha<n$. If $X(t) \in L[0, a]$, then

$\left(D_{P}^{[\alpha, \beta]} I_{N}^{[\alpha, \beta]} X\right)(t)=X(t)$.

Using (4.66), the action of the Pskhu fractional derivative on equation (4.61) gives equation (4.62). 
If $X(t) \in C_{E}^{n-1}$, then

$\left(I_{N}^{[\alpha, \beta]} D_{P}^{[\alpha, \beta]} X\right)(t)=X(t)-\sum_{k=1}^{n} W(\alpha-k, \beta-k, t) \cdot\left(D_{P}^{[\alpha-k, \beta-k]} X\right)(0+)$,

where we use the notation

$\left(D_{P}^{[\alpha, \beta]} X\right)(0+)=\lim _{t \rightarrow 0}\left(D_{P}^{[\alpha, \beta]} X\right)(t)$,

and the function

$W(\alpha, \beta, t):=\frac{1}{(\beta-\alpha) \cdot t} V i(\alpha, \beta, t)=\frac{1}{(\beta-\alpha) \cdot t} \cdot \int_{\alpha}^{\beta} \frac{t^{\xi} d \xi}{\Gamma(\xi)}$.

The function space $C_{E}^{k}$ means the following class of functions

$X(t) \in C_{*}^{k}\left[(0, a) ;-t^{n-\alpha-1} E_{\beta-\alpha}\left[t^{\beta-\alpha} ; n-\alpha\right]\right]$,

where $\mathrm{n}$ is defined by the conditions $n-1<\alpha \leq n$ and $1 \leq k \leq n$ (see Pskhu, A. V. (2005), p. 137). We denote by $C_{*}^{n}[(0, a) ; f(t)]$ the space of functions $X(t) \in L[0, a]$ such that $(X(t) * f(t)) \in C^{n}[0, a]$ and $D^{n}(X(t) * f(t)) \in A C[0, a]$, where $A C[0, a]$ is the space of functions, which are absolutely continuous on $[0, a]$, and the symbol $*$ denoted the convolution

$(f * g)(t):=\int_{0}^{t} f(\tau) \cdot g(t-\tau) d \tau=\int_{0}^{t} f(t-\tau) \cdot g(\tau) d \tau$.

Using (4.67), the action of the Pskhu fractional integral on equation (4.63) gives equation (4.64).

\subsubsection{Duality of UDM Accelerator and NSDM Multiplier}

The accelerator with uniform distributed memory (the UDM accelerator) can be defined by the equation $Y(t)=a([\alpha, \beta]) \cdot D_{N}^{[\alpha, \beta]} X(t)$,

where $D_{N}^{[\alpha, \beta]}$ is the Nakhushev fractional derivative, and $a([\alpha, \beta])$ is the accelerator coefficient, which depends on the interval $[\alpha, \beta]$.

\section{Principle of duality of UDM accelerator and NSDM multiplier:}

$[A \rightarrow M]$ For the concept of accelerator (4.71), there is a dual multiplier, which is the multiplier with non-singular distributed memory (the NSDM multiplier) that is described by the equation

$X(t)=m([\alpha, \beta]) \cdot I_{P}^{[\alpha, \beta]} Y(t)+b_{X}(t)$,

where $I_{P}^{[\alpha, \beta]}$ is the Pskhu fractional derivative of the non-singular distributed order,

$b_{X}(t)=\sum_{k=1}^{n} p_{k}^{P}(0) \cdot t^{\beta-k} \cdot(\alpha-\beta) \cdot E_{\beta-\alpha}\left[t^{\beta-\alpha} ; \beta-k+1\right]$,

and $p_{k}^{P}(0)$ is defined by the expression $p_{k}^{P}(0)=\left(D_{N}^{[\alpha-k, \beta-k]} X\right)(0+)$.

$[M \rightarrow A]$ At the same time, for the NSDM multiplier, which is described by equation

$Y(t)=m([\alpha, \beta]) \cdot I_{P}^{[\alpha, \beta]} X(t)$,

there is a dual concept of the accelerator with uniform distributed memory

$X(t)=a([\alpha, \beta]) \cdot D_{N}^{[\alpha, \beta]} Y(t)$.

In the dual concepts the exogenous and endogenous variables change the roles.

Proof. The proof of this duality is based on Theorem 3 of Pskhu, A. V. (2004) or Theorem 5.1.3 Pskhu, A. V. (2005), p. 142-143, which can be rewritten by the following statement. Let $\beta>\alpha$ and $0<n-1 \leq \beta<n$, where $\mathrm{n}$ is positive integer number. If $X(t) \in L[0, a]$, then

$\left(D_{N}^{[\alpha, \beta]} I_{P}^{[\alpha, \beta]} X\right)(t)=X(t)$.

If $X(t) \in C_{v}^{n-1}$, then

$\left(I_{P}^{[\alpha, \beta]} D_{N}^{[\alpha, \beta]} X\right)(t)=X(t)-\sum_{k=1}^{n} p_{k}^{P}(0) \cdot t^{\beta-k} \cdot(\alpha-\beta) \cdot E_{\beta-\alpha}\left[t^{\beta-\alpha} ; \beta-k+1\right]$,

where

$p_{k}^{P}(0)=\left(D_{N}^{[\alpha-k, \beta-k]} X\right)(0+)$.

The function space $C_{v}^{k}$ means the following class of functions 
$X(t) \in C_{*}^{k}[(0, a) ; W(\alpha-n, \beta-n, t)]$,

where $\mathrm{n}$ is defined by the conditions $n-1<\beta \leq n$ and $1 \leq k \leq n$, Pskhu, A. V. (2005), p. 142.

\subsection{Principle of Decreasing of Fading Parameter for Multiplier with Memory}

The duality of the accelerator and multiplier with memory has been described for the case of equal fading orders. Let us consider a combination of actions of an accelerator with memory and a multiplier with memory, when the fading parameters are different.

Principle of decreasing of fading parameter for multiplier with memory: The action of the accelerator with power-law memory changes the effect of the multiplier with power-law memory by decreasing the parameter of memory fading. Symbolically, this principle can be written in the form of the identity

$E_{0}^{t ; \beta}\left(F_{0}^{t ; \alpha}(X(\tau))\right)=F_{0}^{t ; \alpha-\beta}(X(\tau))$,

which is satisfied for any positive fading parameters $\alpha$ and $\beta$ such that $\alpha>\beta>0$. In other words, the fading parameter of the new multiplier is equal to the difference between the fading parameters of the multiplier and the accelerator.

Equality (4.50) means the following. The action of the multiplier with power-law memory

$Y(t)=F_{0}^{t ; \alpha}(X(\tau))$

and the accelerator with power-law memory

$Z(t)=E_{0}^{t ; \beta}(Y(\tau))$

is equivalent to the action of the multiplier with memory

$Z(t)=F_{0}^{t ; \alpha-\beta}(X(\tau))$

if fading parameters $\alpha$ and $\beta$ satisfy the condition where $\alpha>\beta>0$.

Example 1. The action of the multiplier with power-law memory

$Y(t)=m(\alpha) \cdot I_{R L, 0+}^{\alpha}(X(\tau))$

and the accelerator with power-law memory

$Z(t)=a(\beta) \cdot D_{R L, 0+}^{\beta}(Y(\tau))$

is equivalent to the action of the multiplier with memory

$Z(t)=m(\alpha, \beta) \cdot I_{R L, a^{+}}^{\alpha-\beta}(X(\tau))$

where $m(\alpha, \beta)=m(\alpha) \cdot a(\beta)$ if fading parameters $\alpha$ and $\beta$ satisfy the condition where $\alpha>\beta>0$.

As a result, the fading parameter of the new multiplier is equal to the difference between the fading parameters of the multiplier and the accelerator. The coefficient of the new multiplier is equal to the product of the multiplier and accelerator coefficients.

For this case of the simplest power-law memory, this principle is based on Property 2.2 of Kilbas, A. A., et. al. (2006), p. 74 or Theorem 2.5. of Samko, S. G. et. al. (1993), p. 46. If $\alpha>\beta>0$ and $X(t) \in L_{p}(a, b)$ with $p \geq 1$, then the equality

$D_{R L, a+}^{\beta} I_{R L, a+}^{\alpha} X(t)=I_{R L, a+}^{\alpha-\beta} X(t)$

is valid (see Property 2.2 of Kilbas, A. A., et. al. (2006), p. 74) almost everywhere on $[a, b]$. In particular, if $0<\beta<1$ and $\alpha=1$, then we have

$D_{R L, a+}^{\beta} I_{a^{+}}^{1} X(t)=I_{R L, a+}^{1-\beta} X(t)$.

Example 2. It should be noted that this principle can also be used for the case, when the accelerator is standard. In this case, the fading parameter of the standard accelerator is equal to an integer number (for example, $\beta=1$ ). The proof of this statement is based on the equation, when $\beta=1$ and $\alpha>1$, i.e., the equality

$D^{1} I_{R L, a+}^{\alpha} X(t)=I_{R L, a+}^{\alpha-1} X(t)$

is valid for $\alpha>1$.

Example 3. For the case when the memory of multiplier and accelerator is described by the Liouville fractional integral and derivative, this principle is based on Property 2.12 of Kilbas, A. A., et. al. (2006), p. 89), which states that the equality 
$D_{L,+}^{\beta} I_{L,+}^{\alpha} X(t)=I_{L,+}^{\alpha-\beta} X(t)$

holds for $X(t) \in L_{1}(-\infty,+\infty)$.

\section{Conclusion}

As a result, we got the concepts of multiplier and accelerator with memory, which is a generalization of the standard concepts of the accelerator and the multiplier to the case of economic processes with memory. The equations of the standard accelerator and multiplier are the special cases of the accelerator equation with memory, whose fading is characterized by the one parameter $\alpha \geq 0$. In addition to the standard concepts of the accelerator and the multiplier, the proposed concept of accelerator with memory includes the wide spectrum of intermediate dependencies, which are intermediate between the standard accelerators and the standard multiplier. The proposed approach allows us to take into account the fact that economic agents have a memory of the previous changes of the factor and indicator on a finite time interval. An important property of the suggested new concepts is the duality of the concepts of the accelerator with memory and the multiplier with memory.

The proposed concepts of the accelerator with memory and the multiplier with memory allow us to build new macroeconomic models that take into account the wide class of memory effects, and to obtain solutions for the corresponding equations by using the mathematical tools of derivatives and integrals of non-integer orders.

\section{References}

1. Allen, R. G. D. (1959). Mathematical Economics. Second Edition. London: Macmillan 1959. 812 p.

2. Allen, R. G. D. (1968). Macro-economic theory. A Mathematical Treatment. London: Macmillan, 1968. 420 p.

3. Almeida, R. (2017). A Caputo fractional derivative of a function with respect to another function // Communications in Nonlinear Science and Numerical Simulation. 2017. Vol. 44. P. 460-481. DOI: 10.1016/j.cnsns.2016.09.006 (arXiv:1609.04775)

4. Bagley, R. L., Torvik, P. J. (2000a) On the existence of the order domain and the solution of distributed order equations - Part I // International Journal of Applied Mathematics. 2000. Vol. 2. P.865-882.

5. Bagley R. L., Torvik P. J. (2000b) On the existence of the order domain and the solution of distributed order equations - Part II // International Journal of Applied Mathematics. 2000. Vol. 2. P. 965-987.

6. Baker, C. T. H. (2000). Retarded differential equations // Journal of Computational and Applied Mathematics. 2000. Vol. 125. No. 1-2. P. 309-335.

7. Bernanke, B., Gertler, M., Gilchrist, S. (1996). The financial accelerator and the flight to quality // Review of Economics and Statistics. 1996. Vol. 78. No. 1. P. 1-15

8. Caputo, M. (1995). Mean fractional-order-derivatives differential equations and filters // Annali dell'Universita di Ferrara. 1995. Vol. 41. No. 1. P. 73-84. DOI: 10.1007/BF02826009

9. Caputo, M. (2001). Distributed order differential equations modelling dielectric induction and diffusion // Fractional Calculus and Applied Analysis. 2001. Vol. 4. No. 4. P. 421-442.

10. Clark, J. M. (1917). Business acceleration and the law of demand: A Technical factor in economic cycles // Journal of Political Economy. 1917. Vol. 25. No. 3. P. 217-235.

11. Diethelm, K. (2010). The Analysis of Fractional Differential Equations: An Application-Oriented Exposition Using Differential Operators of Caputo Type. Berlin: Springer-Verlag, 2010. 247 p. DOI: 10.1007/978-3-642-14574-2

12. Elsgolts, L. E., Norkin, S. B. (1973). Introduction to the Theory and Application of Differential Equations with Deviating Arguments. New York: Academic Press, 1973. 356p.

13. Gabaix, X. (2009) Power laws in economics and finance // Annual Review of Economics. 2009. Vol. 1. No. 1. P. 255-293.

14. Gabaix, X. (2016). Power laws in economics: An introduction // Journal of Economic Perspectives. 2016. Vol. 30. No. 1. P. $185-206$.

15. Galiullin, A. S. (1984). Inverse Problems of Dynamics. Moscow: Mir Publishers, 1984. 149p.

16. Gladwell, G. M. L., Morassi, A. (2011). (Eds.). Dynamical Inverse Problems: Theory and Application. Wien: Springer-Verlag, 2011. 226p. DOI: 10.1007/978-3-7091-0696-9

17. Jiao, Z., Chen, Y. Q., Podlubny, I. (2012). Distributed-Order Dynamic Systems: Stability, Simulation, Applications and Perspectives. London: Springer, 2012. 90 p. DOI: 10.1007/978-1-4471-2852-6

18. Kahn, R. F. (1931). The relation of home investment to unemployment // The Economic Journal. 1931. Vol. 41. No. 162. P. 173-198. DOI: $10.2307 / 2223697$

19. Keynes, J. M. (1936). The General Theory of Employment, Interest and Money. New York: Harcourt, Brace and Company, 1936. (Cambridge: Cambridge University Press, 2012. 451 p.)

20. Keynes, J. M. (2012). The Collected Writings of John Maynard Keynes, Volume VII: The General Theory of Employment, Interest and Money // Edited by E. Johnson, D.E. Moggridge. Cambridge University Press, 2012. 451 p.

21. Kilbas, A .A., Srivastava, H. M., Trujillo, J. J. (2006). Theory and Applications of Fractional Differential Equations. Amsterdam: Elsevier, 2006. 540 p. ISBN: 9780444518323 
22. Kiryakova, V. (1994). Generalized Fractional Calculus and Applications. New York: Longman and J. Wiley, 1994. $360 \mathrm{p}$.

23. Kiryakova, V. (2008). A brief story about the operators of the generalized fractional calculus // Fractional Calculus and Applied Analysis. 2008. Vol. 11. No. 2. P. 203-220.

24. Kiryakova, V., Luchko, Yu. (2013). Riemann-Liouville and Caputo type multiple Erdelyi-Kober operators // Central European Journal of Physics. 2013. Vol. 11. No. 10. P. 1314-1336.

25. Lokshin, A. A., Suvorova, Yu. V. (1982). Mathematical Theory of Wave Propagation in Media with Memory. Moscow: Moscow State University. 1982. 152 p. [in Russian]

26. Lorenzo, C. F., Hartley, T. T. (2002) Variable order and distributed order fractional operators // Nonlinear Dynamics. 2002. Vol. 29. No. 1. P. 57-98. DOI: 10.1023/A:1016586905654

27. Luchko, Yu., Trujillo, J. J. (2007). Caputo-type modification of the Erdelyi-Kober fractional derivative // Fractional Calculus and Applied Analysis. 2007. Vol. 10. No. 3. P. 249-268.

28. Mainardi, F. (2010). Fractional Calculus and Waves Linear Viscoelasticity: An Introduction to Mathematical Models. London: Imperial College Press, 2010. 368 p.

29. Nakhushev, A. M. (1998). On the positivity of continuous and discrete differentiation and integration operators that are very important in fractional calculus and in the theory of equations of mixed type // Differential Equations. 1998. Vol. 34. No. 1. P. 103-112.

30. Nakhushev, A. M. (2003). Fractional Calculus and its Application. Moscow: Fizmatlit, 2003. 272 p. [in Russian]

31. Podlubny, I. (1998). Fractional Differential Equations. San Diego: Academic Press, 1998. 340 p.

32. Prudnikov, A. P., Brychkov, Yu. A., Marichev O.I. (1986). Integrals and Series: Elementary Functions. Vol. 1. New York: Gordon and Breach, 1986. 798 p.

33. Pskhu, A. V. (2004). On the theory of the continual integro-differentiation operator // Differential Equations. 2004. Vol. 40. No. 1. P. 128-136. DOI: 10.1023/B:DIEQ.0000028722.41328.21

34. Pskhu, A. V. (2005). Partial Differential Equations of Fractional Order. Moscow: Nauka, 2005. 199 p. [in Russian]

35. Samko, S. G., Kilbas, A. A., Marichev, O. I. (1993). Fractional Integrals and Derivatives Theory and Applications. New York: Gordon and Breach, 1993. 1006 p.

36. Sverdlove R. (1981). Inverse problems for dynamical systems // Journal of Differential Equations. 1981. Vol. 42. No. 1. P. 72-105. DOI: 10.1016/0022-0396(81)90034-6

37. Tarasov, V. E. (2010). Fractional Dynamics: Applications of Fractional Calculus to Dynamics of Particles, Fields and Media. New York: Springer, 2010. 505 p. DOI: 10.1007/978-3-642-14003-7

38. Tarasov, V. E. (2013). No violation of the Leibniz rule. No fractional derivative // Communications in Nonlinear Science and Numerical Simulation. 2013. Vol. 18. No. 11. P. 2945-2948. DOI: 10.1016/j.cnsns.2013.04.001 (arXiv:1402.7161)

39. Tarasov, V. E. (2016a). Leibniz rule and fractional derivatives of power functions // Journal of Computational and Nonlinear Dynamics. 2016. Vol. 11. No. 3. Article ID: 031014. DOI: 10.1115/1.4031364

40. Tarasov, V. E. (2016b). On chain rule for fractional derivatives // Communications in Nonlinear Science and Numerical Simulation. Vol. 30. No. 1-3. (2016) P. 1-4. DOI: 10.1016/j.cnsns.2015.06.007

41. Tarasov, V. E., Tarasova, V. V. (2016). Long and short memory in economics: fractional-order difference and differentiation // IRA-International Journal of Management and Social Sciences. 2016. Vol. 5. No. 2. P. 327-334. DOI: $10.21013 /$ jmss.v5.n2.p10

42. Tarasov, V. E., Tarasova, V. V. (2017). Time-dependent fractional dynamics with memory in quantum and economic physics // Annals of Physics. 2017. DOI: 10.1016/j.aop.2017.05.017

43. Tarasova, V. V., Tarasov, V. E. (2016a). Economic accelerator with memory: discrete time approach // Problems of Modern Science and Education [Problemy Sovremennoj Nauki i Obrazovaniya]. 2016. No. 36 (78). P. 37-42. DOI: 10.20861/2304-2338-2016-78-002

44. Tarasova, V. V., Tarasov, V. E. (2016b). Elasticity for economic processes with memory: fractional differential calculus approach // Fractional Differential Calculus. 2016. Vol. 6. No. 2. P. 219-232. DOI: 10.7153/fdc-06-14

45. Tarasova, V. V., Tarasov, V. E. (2016c). Fractional dynamics of natural growth and memory effect in economics // European Research. 2016. No. 12 (23). P. 30-37. DOI: 10.20861/2410-2873-2016-23-004

46. Tarasova, V. V., Tarasov, V. E. (2016d). Hereditary generalization of Harrod-Domar model and memory effects // Journal of Economy and Entrepreneurship [Ekonomika i Predprinimatelstvo]. 2016. No. 10-2 (75-2). P. 72-78. [in Russian]

47. Tarasova, V. V., Tarasov, V. E. (2016e). Influence of memory effects on world economics and business // Azimuth Scientific Research: Economics and Management [Azimut Nauchnih Issledovanii: Ekonomika i Upravlenie]. 2016. Vol. 5. No. 4 (17). P. 369-372. [in Russian]

48. Tarasova, V. V., Tarasov, V. E. (2016f). Keynesian model of economic growth with memory // Economics and Management: Problems, Solutions [Ekonomika i Upravlenie: Problemy i Resheniya]. 2016. No. 10-2 (58). P. 21-29. [in Russian]

49. Tarasova, V. V., Tarasov, V. E. (2016g). Memory effects in hereditary Harrod-Domar model // Problems of Modern Science and Education [Problemy Sovremennoj Nauki i Obrazovaniya]. 2016. No. 32 (74). P. 38-44. [in Russian] DOI: 10.20861/2304-2338-2016-74-002

50. Tarasova, V. V., Tarasov, V. E. (2016h). Memory effects in hereditary Keynes model // Problems of Modern Science and Education [Problemy Sovremennoj Nauki i Obrazovaniya]. 2016. No. 38 (80). P. 56-61. [in Russian] DOI: 10.20861/2304-2338-2016-80-001 
51. Tarasova, V. V., Tarasov, V. E. (2017a). Dynamic intersectoral models with memory that generalize Leontief model // Journal of Economy and Entrepreneurship [Ekonomika i Predprinimatelstvo]. 2017. No. 2-1 (79-1). P. 913-924. [in Russian]

52. Tarasova, V. V., Tarasov, V. E. (2017b). Economic growth model with constant pace and dynamic memory // Problems of Modern Science and Education. 2017. No. 2 (84). P. 40-45. DOI: 10.20861/2304-2338-2017-84-001

53. Tarasova, V. V., Tarasov, V. E. (2017c). Economic interpretation of fractional derivatives // Progress in Fractional Differentiation and Applications. 2017. Vol. 3. No. 1. P. 1-7. DOI: 10.18576/pfda/030101

54. Tarasova, V. V., Tarasov, V. E. (2017d). Logistic map with memory from economic model // Chaos, Solitons and Fractals. 2017. Vol. 95. P. 84-91. DOI: 10.1016/j.chaos.2016.12.012

55. Tarasova, V. V., Tarasov, V. E. (2017e). Macroeconomic models with dynamic memory // Journal of Economy and Entrepreneurship [Ekonomika i Predprinimatelstvo]. 2017. No. 3-2 (80-2). P. 26-35. [in Russian]

56. Tarasova, V. V., Tarasov, V. E. (2018a). Concept of dynamic memory in economics // Communications in Nonlinear Science and Numerical Simulation. 2018. Vol. 55. P. 127-145. DOI: 10.1016/j.cnsns.2017.06.032

57. Tarasova, V. V., Tarasov, V. E. (2018b). Dynamic intersectoral models with power-law memory // Communications in Nonlinear Science and Numerical Simulation. 2018. Vol. 54. P. 100-117. DOI: 10.1016/j.cnsns.2017.05.015

58. Teyssiere, G., Kirman, A. P. (2007). Long Memory in Economics. Berlin, Heidelberg: Springer-Verlag, 2007. 390 p.

59. Volterra, V. (2005). Theory of Functionals and of Integral and Integro-Differential Equations. Dover, 2005. 299 p. 